University of Nebraska - Lincoln

DigitalCommons@University of Nebraska - Lincoln

Faculty Publications, Department of Mathematics

Mathematics, Department of

$1-1-1999$

\title{
Lack of Time-Delay Robustness for Stabilization of a Structural Acoustics Model
}

\author{
George Avalos \\ University of Nebraska-Lincoln, gavalos@math.unl.edu \\ Irena Lasiecka \\ University of Virginia \\ Richard Rebarber \\ University of Nebraska-Lincoln, rrebarber1@unl.edu
}

Follow this and additional works at: https://digitalcommons.unl.edu/mathfacpub

Part of the Mathematics Commons

Avalos, George; Lasiecka, Irena; and Rebarber, Richard, "Lack of Time-Delay Robustness for Stabilization of a Structural Acoustics Model" (1999). Faculty Publications, Department of Mathematics. 2.

https://digitalcommons.unl.edu/mathfacpub/2

This Article is brought to you for free and open access by the Mathematics, Department of at DigitalCommons@University of Nebraska - Lincoln. It has been accepted for inclusion in Faculty Publications, Department of Mathematics by an authorized administrator of DigitalCommons@University of Nebraska - Lincoln. 


\title{
LACK OF TIME-DELAY ROBUSTNESS FOR STABILIZATION OF A STRUCTURAL ACOUSTICS MODEL*
}

\author{
GEORGE AVALOS ${ }^{\dagger}$, IRENA LASIECKA IND RICHARD REBARBER $^{\S}$
}

\begin{abstract}
In this paper we consider a natural robustness question for a model for structural acoustics. This model, which has been of great interest in recent years, is represented by a wave equation in $\mathbb{R}^{2}$ coupled to a Kelvin-Voigt beam; the coupling is natural physically, and is represented mathematically by highly unbounded operators. We assume that the observation consists of point evaluation of the beam position, the beam velocity, and the wave velocity. We are interested in the effect of arbitrarily small delays in the feedback loop on a controller that uses these observations. We show that it is not possible to construct a dynamic stabilizer of a very general form-including static feedback - such that the stabilization is robust with respect to delays in the feedback loop. In order to do this we need to carefully analyze the input-to-output map. Finally, we relate these results to already existing numerical results obtained for a Galerkin approximation of the system.
\end{abstract}

Key words. time delays, robust stabilization, coupled partial differential equations, transfer functions, dynamic stabilization, structural acoustics

AMS subject classifications. 93C20, 93D09, 93D15, 93D25, 35M10

PII. S0363012997331135

1. Introduction. In this paper we consider a natural robustness question for a model for structural acoustics. This model has been of great interest in recent years (see Banks et al. [6, 7, 8, 9, 10, 11, 12] and the references therein, Avalos [1], and Avalos and Lasiecka $[2,3])$. In this introduction we roughly describe the problem and our results, and we leave the precise problem description and technical details for later sections.

Most of the mathematical analysis of this structural acoustics model has been done with a two-dimensional approximation; see $[6,7,12]$ for details about the modeling for this system. We represent an acoustic cavity by a rectangular region in $\mathbb{R}^{2}$ and we consider one side of the boundary to be a flexible beam and the other three sides to be hard walls. Sound waves inside the cavity are described by a wave equation. The displacement of the flexible beam, which we call the active boundary, is described by a beam equation with Kelvin-Voigt damping. The coupling of these two equations is natural physically and is represented mathematically by highly unbounded operators. In the references above the control is formed by using piezoceramic patches on the active boundary, which produce moment forces when a voltage is applied. The control goal we consider here is stabilization of the system, in particular the attenuation of the acoustic pressure in the cavity. This attenuation might be described by exponential stability of an appropriate state space model, or by input-output stability of an appropriate input-output model.

* Received by the editors December 5, 1997; accepted for publication (in revised form) December 2, 1998; published electronically July 22, 1999.

http://www.siam.org/journals/sicon/37-5/33113.html

${ }^{\dagger}$ Department of Mathematics, Texas Tech University, Lubbock, TX 79409 (avalos@me.ttu.edu). The work of this author was partially supported by NSF grant DMS-9710981.

${ }^{\ddagger}$ Department of Applied Mathematics, Thornton Hall, University of Virginia, Charlottesville, VA 22903 (il2v@amsun.apma.virginia.edu). The work of this author was partially supported by NSF grant DMS-9504822 and ARO grant MA DAAAHO4-96-0059.

${ }^{\S}$ Department of Mathematics and Statistics, University of Nebraska-Lincoln, Lincoln, NE 685880323 (rrebarbe@math.unl.edu). The work of this author was partially supported by NSF grant DMS-9623392. 
In this paper we assume that the full state of the system is not available for use by the controller and that we have access to the following observations of the system: point evaluation of the beam displacement at some point or points on the beam, point evaluation of the beam velocity at some point or points on the beam, and point evaluation of the acoustic pressure at some points in the cavity. We consider controls determined by a dynamic compensator that uses these observations as its input.

We are interested in the effect of arbitrarily small delays in the feedback loop on the stability of the closed-loop system. Roughly speaking, we say that feedback stabilization of a system is robust with respect to delays if delays introduced into the feedback loop do not destroy the stability provided that the delays are sufficiently small. In this paper we answer the following question: Is it possible to construct a dynamic stabilizer (or a static feedback) for this structural acoustics systems such that the stabilization is robust with respect to small delays in the feedback loop?

For a large class of dynamic stabilizers and static feedbacks we show that the answer to this question is negative, since we find that there exists a sequence of delays $\left\{\varepsilon_{j}\right\}$ such that $\varepsilon_{j} \rightarrow 0$ and the closed-loop system with delay $\varepsilon_{j}$ has an unstable transfer function pole. See section 5 for precise statements of the relevant results. In Banks, Demetriou, and Smith [8] the effect of delays on an $H^{\infty}$ dynamic compensator for this system is studied numerically. In particular, in [8] delays are inserted in three places in the feedback loop, and the robustness with respect to these delays is studied for a Galerkin approximation of the closed-loop system. In section 6 we adapt our results to the setup in [8] and give a heuristic explanation for the results in [8] based on a frequency domain analysis.

The approach we use for these lack-of-robustness results is systems theoretic in the sense that we first show that our system is in a particular class of systems and then appeal to theorems about this class. The class in question is the class of regular systems, which is a very general class whose basic properties were developed by Weiss in [25] and [26], and for which there is now a substantial amount of control machinery; see for instance $[17,18,19,21,22,23,27,29]$. In order to show that our system is regular when the observation includes point observations of the beam displacement and velocity, but does not include point observations of the acoustic pressure, we rely heavily on results by Avalos and Lasiecka [2] and Avalos [1]. When the observation includes point evaluation of acoustic pressure, proving regularity involves a careful analysis of the map from control to observation. This regularity is of interest independent of robustness questions, since many other control questions, e.g., adaptive control [18], stability radii [19], or dynamic stabilization [27], can be studied in the regular systems framework.

In the case when the observation does not include point evaluation of acoustic pressure, we have a natural $(A, B, C, D)$ state space realization for the system, and our results can be stated in terms of exponential stability. In the case where the observation includes point evaluation of acoustic pressure, the state space that accommodates both the control and observation does not coincide with the basic energy space - it requires $(1 / 2)$ more derivatives in the wave variable - so we present our results in input-output form.

The paper is organized as follows. In section 2 we present the controlled, observed structural acoustics system. In section 3 we show that when the observation is point evaluation of acoustic pressure, then the input-to-output map is well posed, that is, for any $T>0$ this map is in $\mathcal{L}\left(L^{2}[0, T ; U], L^{2}[0, T ; Y]\right)$, where $U$ is the control space and $Y$ is the observation space. In section 4 we prove regularity of the system. In 
section 5 we discuss some results on robustness and lack of robustness with respect to delays, which can be immediately applied to our system. In section 6 we relate and compare our results to the numerical studies in [8]. In all the sections except section 3 , we allow the acoustic cavity to be either a rectangle or any region in $\mathbb{R}^{2}$ with a smooth boundary. In section 3 we require the cavity to be a rectangle. In [5] the results in section 3 are obtained, using very different techniques, when $\Omega$ is a region in $\mathbb{R}^{2}$ or $\mathbb{R}^{3}$ with smooth boundary.

2. The controlled, observed structural acoustics system. In this section we present and analyze the partial differential equation model. This model is based on the one in $[9,12]$, but we will use a scaled, slightly abstracted version. Let $\Omega$ be either a rectangular region in $\mathbb{R}^{2}$ or a region in $\mathbb{R}^{2}$ with Lipschitz boundary $\Gamma$. Let $\Gamma_{0}$ be a smooth $\left(C^{2}\right)$ segment of $\Gamma$ with endpoints $a$ and $b$. Let $z=z(t, x)$ for $t \in[0, \infty)$ and $x \in \Omega$, let $v=v(t, \xi)$ for $t \in[0, \infty)$ and $\xi \in \Gamma_{0}$, and let $\partial / \partial \nu$ denote the outward normal derivative. Let $U=\mathbb{R}^{r}$ and $B \in \mathcal{L}\left(U, H^{-\alpha}\left(\Gamma_{0}\right)\right)$, where $\alpha$ will be specified throughout to be

$$
\begin{aligned}
& \alpha=\frac{7}{4} \text { when } \Omega \text { is rectangular } \\
& \text { and } \alpha=\frac{5}{3} \text { when } \Omega \text { has a smooth boundary. }
\end{aligned}
$$

We refer to the following as the structural acoustics model:

$$
\begin{aligned}
z_{t t} & =\Delta z \text { on }[0, \infty) \times \Omega, \\
\frac{\partial z}{\partial \nu} & =v_{t} \text { on }[0, \infty) \times \Gamma_{0}, \\
\frac{\partial z}{\partial \nu} & =0 \text { on }[0, \infty) \times \Gamma \backslash \Gamma_{0}, \\
v_{t t} & =-\Delta^{2} v-\Delta^{2} v_{t}-z_{t}+B u \text { on }[0, \infty) \times \Gamma_{0}, \\
v(a, t) & =v_{t}(b, t)=\frac{\partial v(a, t)}{\partial x}=\frac{\partial v(b, t)}{\partial x}=0 \quad \forall t \in[0, \infty) .
\end{aligned}
$$

The model discussed in $[6,7,8,9,10,11,12]$, suitably scaled, is a special case of (2.2): in this case $\Omega$ is a rectangular region, $\Gamma_{0}$ is the bottom side of the rectangle, and $B$ is of the form

$$
B=\sum_{i=1}^{r} \alpha_{i} \delta^{\prime}\left(\eta_{i}\right),
$$

where $\delta^{\prime}\left(\eta_{i}\right)$ are derivatives of delta functions evaluated at $\eta_{i} \in \Gamma_{0}$ and $\alpha_{i} \in \mathbb{R}$. The physical interpretation for this particular control operator is that its control action is realized by the strategic placement of piezoelectric ceramic patches on the (flexible) boundary $\Gamma_{0}$; a voltage is subsequently applied through these patches and the resulting bending moments can be interpreted as derivatives of delta functions.

The observation $y(t)$ considered in [6] and [8] is a vector with components of the form $v\left(t, \xi_{0}\right)$ for $\xi_{0} \in \Gamma_{0}, v_{t}\left(t, \xi_{0}\right)$ for $\xi_{0} \in \Gamma_{0}$, and $z_{t}\left(t, x_{0}\right)$ for $x_{0} \in \bar{\Omega}$; these 
observations are chosen because they are likely to be physically observable. The acoustic pressure is proportional to $z_{t}$, so we often refer to an observation of $z_{t}$ as an observation of acoustic pressure.

In [1], [2] the control system (2.2) was put into the following state-space form. Define the operator $A: L^{2}(\Omega) \supset \mathcal{D}(A) \rightarrow L^{2}(\Omega)$ by $A=-\Delta$ with

$$
\mathcal{D}(A)=\left\{z \in L^{2}(\Omega) / \mathbb{R} \cap H^{2}(\Omega) \mid \frac{\partial z}{\partial \nu}=0 \text { on } \Gamma\right\},
$$

where $L^{2}(\Omega) / \mathbb{R}=\left\{f \in L^{2}(\Omega) \mid \int_{\Omega} f=0\right\} ;$ viz, $L^{2}(\Omega) / \mathbb{R}$ is the orthogonal complement of the space of constant functions in $L^{2}(\Omega)$. $A$ is symmetric positive definite on $L^{2}(\Omega)$, so fractional powers of $A$ are well defined. In particular, from [14] we have

$$
\mathcal{D}\left(A^{\beta / 2}\right)=L^{2}(\Omega) / \mathbb{R} \cap H^{\beta}(\Omega) \quad \text { for } \beta \in\left[0, \frac{3}{2}\right) .
$$

Define $\AA: L^{2}\left(\Gamma_{0}\right) \supset \mathcal{D}(\AA) \rightarrow L^{2}\left(\Gamma_{0}\right)$ by $\AA=\Delta^{2}$ with

$$
\mathcal{D}(\AA)=H^{4}\left(\Gamma_{0}\right) \cap H_{0}^{2}\left(\Gamma_{0}\right) .
$$

$\AA$ is symmetric positive definite on $L^{2}\left(\Gamma_{0}\right)$, so its fractional powers are well defined. In particular, by [14] we have

$$
\mathcal{D}\left(\AA^{\beta / 4}\right)=H_{0}^{\beta}\left(\Gamma_{0}\right) \quad \text { for } \beta \in\left[0, \frac{5}{2}\right) .
$$

Since $\AA$ is symmetric positive definite on a Hilbert space and the dual space of $H_{0}^{\beta}$ is $H^{-\beta}$, we see that

$$
\mathcal{D}\left(\AA^{\beta / 4}\right)^{\prime}=H^{-\beta}\left(\Gamma_{0}\right) \quad \text { for } \beta \in\left[0, \frac{5}{2}\right) .
$$

Define

$$
H_{1}=\mathcal{D}\left(A^{\frac{1}{2}}\right) \times L^{2}(\Omega)=\left(L^{2}(\Omega) / \mathbb{R} \cap H^{1}(\Omega)\right) \times L^{2}(\Omega)
$$

and

$$
H_{0}=\mathcal{D}\left(\AA^{\frac{1}{2}}\right) \times L^{2}\left(\Gamma_{0}\right)=H_{0}^{2}\left(\Gamma_{0}\right) \times L^{2}\left(\Gamma_{0}\right) .
$$

Let $A_{1}: H_{1} \supset \mathcal{D}\left(A_{1}\right) \rightarrow H_{1}$ and $A_{0}: H_{0} \supset \mathcal{D}\left(A_{0}\right) \rightarrow H_{0}$ be defined by

$$
\begin{aligned}
A_{1} & :=\left[\begin{array}{cc}
0 & I \\
-A & 0
\end{array}\right] \quad \text { with } \\
D\left(A_{1}\right) & =\left\{\left[z_{1}, z_{2}\right]^{T} \in D(A) \times D\left(A^{\frac{1}{2}}\right)\right\}
\end{aligned}
$$

and

$$
\begin{aligned}
A_{0} & :=\left[\begin{array}{cc}
0 & I \\
-\AA & -\AA
\end{array}\right] \text { with } \\
D\left(A_{0}\right) & =\left\{\left[v_{1}, v_{2}\right]^{T} \in\left[D\left(\AA^{\frac{1}{2}}\right)\right]^{2} \ni v_{1}+v_{2} \in D(\AA)\right\} .
\end{aligned}
$$


We define the Neumann map $N$ on $H^{s}(\Gamma)$ for $s>-1 / 2$ by setting $N g:=z$ for $g \in H^{s}(\Gamma)$, where $z$ is the unique solution in $L^{2}(\Omega) / \mathbb{R}$ of the equation

$$
\langle A z, v\rangle_{\left[D\left(A^{\frac{1}{2}}\right)\right]^{\prime} \times D\left(A^{\frac{1}{2}}\right)}=\int_{\Omega} \nabla z \cdot \nabla v=\langle g, v\rangle_{H^{s}(\Gamma) \times\left[H^{s}(\Gamma)\right]^{\prime}}
$$

for all $v \in D\left(A^{\frac{1}{2}}\right)$. From [14], we have that

$$
N \in \mathcal{L}\left(L^{2}\left(\Gamma_{0}\right), \mathcal{D}\left(A^{\frac{3}{4}-\epsilon}\right)\right) \text { for arbitrary } \epsilon>0,
$$

and this boundedness further implies that

$$
A N \in \mathcal{L}\left(L^{2}\left(\Gamma_{0}\right),\left[\mathcal{D}\left(A^{\frac{1}{4}+\epsilon}\right)\right]^{\prime}\right) \text { for arbitrary } \epsilon>0 .
$$

We also define $\gamma: H^{1}(\Omega) \rightarrow H^{\frac{1}{2}}\left(\Gamma_{0}\right)$ by $\gamma(z)=\left.z\right|_{\Gamma_{0}}$ and

$$
C=\left[\begin{array}{cc}
0 & 0 \\
0 & \gamma^{*}
\end{array}\right] \text {, }
$$

so $C \in \mathcal{L}\left(H_{0},\{0\} \times\left[D\left(A^{\frac{1}{2}}\right)\right]^{\prime}\right)$. Let $\mathcal{X}=H_{1} \times H_{0}$; we refer to the usual product norm on $\mathcal{X}$ by $\|\cdot\|$, and other norms will be indicated by an appropriate subscript. Now define $\mathcal{A}: \mathcal{D}(\mathcal{A}) \supset \mathcal{X} \rightarrow \mathcal{X}$ by

$$
\mathcal{A}:=\left[\begin{array}{cc}
A_{1} & C \\
-C^{*} & A_{0}
\end{array}\right]
$$

with

$$
\begin{aligned}
D(\mathcal{A})= & \left\{\left[z_{1}, z_{2}, v_{1}, v_{2}\right]^{T} \in\left[D\left(A^{\frac{1}{2}}\right)\right]^{2} \times\left[D\left(\AA^{\frac{1}{2}}\right)\right]^{2}\right. \text { such that } \\
& \left.-z_{1}+N v_{2} \in D(A) \text { and such that } v_{1}+v_{2} \in D(\AA)\right\} .
\end{aligned}
$$

Let

$$
X(t)=\left[z(t), z_{t}(t), v(t), v_{t}(t)\right]^{T} .
$$

If $u(t) \equiv 0$, then $(2.2)$ is formally equivalent to

$$
\dot{X}(t)=\mathcal{A} X(t) .
$$

It is shown in Theorem 1.1 in [1] that $\mathcal{A}$ is the generator of a strongly continuous semigroup $S(t)$ on $\mathcal{X}$; see also Banks and Smith [11].

Let $R(s, \mathcal{A})=(s I-\mathcal{A})^{-1}$. For $\alpha \in \mathbb{R}$, let $\mathbb{C}_{\alpha}:=\{s \in \mathbb{C} \mid \boldsymbol{\operatorname { R e }}(s)>\alpha\}$. The system under consideration in this paper differs from the system in [3] in that the wave equation here has Neumann conditions on $\Gamma \backslash \Gamma_{0}$, while the wave equation in [3] has Dirichlet conditions. The arguments in [3] can be easily modified to show that $S(t)$ is not exponentially stable, so Proposition 2 in Prüss [20] implies that

$$
\sup _{s \in \mathbb{C}_{0}}\|R(s, \mathcal{A})\|_{\mathcal{L}(\mathcal{X})}=\infty
$$


The arguments in [3] can also be modified to show that $S(t)$ is strongly stable and that

$$
\sigma(\mathcal{A}) \cap i \mathbb{R}=\emptyset,
$$

where $\sigma(\mathcal{A})$ is the spectrum of $\mathcal{A}$. Let $s \in i \mathbb{R}$. If $\left\{s_{n}\right\} \subset \overline{\mathbb{C}}_{0}, s=\lim _{n \rightarrow \infty} s_{n}$, and $\left\{\left\|R\left(s_{n}, \mathcal{A}\right)\right\|\right\}$ is unbounded, then $s \in \sigma(\mathcal{A})$, which is in contradiction to (2.11). Hence the only way $(2.10)$ can be true is if

$$
\limsup _{|s| \rightarrow \infty, s \in \mathbb{C}_{0}}\|R(s, \mathcal{A})\|_{\mathcal{L}(\mathcal{X})}=\infty .
$$

Condition (2.12) is important for our lack-of-robustness results.

Let

$$
\mathcal{B}=[0,0,0, B]^{T} \in \mathcal{L}\left(U,\{0\}^{3} \times H^{-\alpha}\left(\Gamma_{0}\right)\right) .
$$

Then (2.2) is formally equivalent to

$$
\dot{X}(t)=\mathcal{A} X(t)+\mathcal{B} u(t),
$$

which holds pointwise in time in

$$
X_{-1}:=\left[\mathcal{D}\left(\mathcal{A}^{*}\right)\right]^{\prime} .
$$

$S(t)$ extends to a semigroup on $\left[\mathcal{D}\left(\mathcal{A}^{*}\right)\right]^{\prime}$ and $(2.2)$ is also formally equivalent to

$$
\begin{aligned}
& z_{t t}=-A z+A N v_{t},
\end{aligned}
$$

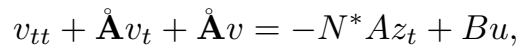

which holds pointwise in time in $[\mathcal{D}(A)]^{\prime}$ and $[\mathcal{D}(\AA)]^{\prime}$, respectively.

We now discuss the observations of this system. Let $C_{\eta}$ be a point evaluation at $\eta$; we will use the same notation when $\eta \in \Gamma_{0}$ or when $\eta \in \bar{\Omega}$. Let $\xi_{0} \in \Gamma_{0}$ and $x_{0} \in \bar{\Omega}$. We define the following operators on $\mathcal{X}$ by

$$
\mathcal{C}_{1}:=\left[0,0, C_{\xi_{0}}, 0\right], \quad \mathcal{C}_{2}:=\left[0,0,0, C_{\xi_{0}}\right], \quad \mathcal{C}_{3}:=\left[0, C_{x_{0}}, 0,0\right] .
$$

We are ultimately interested in observations that contain several terms of this type, but it is most convenient to analyze these separately; in particular, our analysis of $\mathcal{C}_{3}$ necessarily will be much different (and more difficult) than our analysis of $\mathcal{C}_{1}$ or $\mathcal{C}_{2}$.

We wish to show that (2.2) with any of these observations is a regular system. For a detailed discussion of regular systems, see Weiss [25, 26]. Definition 2.1 given below for a well-posed, controlled, observed system is not as detailed as that given in [25], but it is equivalent when the system is given by a boundary-controlled partial differential equation of the type we are currently considering.

Definition 2.1. Let $\mathcal{X}, Y$, and $U$ be Hilbert spaces. A system with state $X(t) \in$ $\mathcal{X}$, input $u(t) \in U$, and observation $y(t) \in Y$ is well posed if for some (and hence all) $T>0$,

(1) $X(t)=S(t) X(0)$ for a strongly continuous semigroup $S(t)$ when $u(t) \equiv 0$;

(2) The map $u(\cdot) \rightarrow X(T)$ is bounded from $L^{2}[0, T ; U]$ into $\mathcal{X}$;

(3) The map $X(0) \rightarrow y(\cdot)$ is bounded from $\mathcal{X}$ into $L^{2}[0, T ; Y]$ when $u(t) \equiv 0$; 
(4) The map $\mathcal{T}: u(\cdot) \rightarrow y(\cdot)$ with $X(0)=0$ is bounded from $L^{2}[0, T ; U]$ into $L^{2}[0, T ; Y]$.

For many purposes we need only be concerned with the input-output map $\mathcal{T}$.

DeFINITION 2.2. If an input-output map $\mathcal{T}$ satisfies condition (4), we say that it is a well-posed input-output system.

If the controlled system is formally represented by (2.13) and condition (2) of Definition 2.1 is satisfied, then we say that $\mathcal{B}$ is an admissible control (input) operator for $S(t)$. If the uncontrolled system with observation is formally represented by $(2.9)$ and

$$
y(t)=\mathcal{C} X(t)
$$

and condition (3) of Definition 2.1 is satisfied, then we say that $\mathcal{C}$ is an admissible observation (output) operator for $S(t)$. From Proposition 3.2 in [25], condition (4) in Definition 2.1 implies that if

$$
L_{\alpha}^{2}[0, \infty, U]:=\left\{f \in L_{\mathrm{loc}}^{2}[0, \infty ; U] \mid \int_{0}^{\infty}\|f(t)\|_{U}^{2} e^{-2 \alpha t} d t<\infty\right\},
$$

then there exists $\beta \in \mathbb{R}$ such that

$$
\mathcal{T} \in \mathcal{L}\left(L_{\beta}^{2}[0, \infty ; U), L_{\beta}^{2}[0, \infty ; Y)\right) .
$$

While the well-posedness of the system with the observation operator $\mathcal{C}_{1}$ follows from basic Sobolev embeddings and the interior smoothness guaranteed by the statespace topology, this is not the case for the observation operators $\mathcal{C}_{2}$ and $\mathcal{C}_{3}$. Indeed, the topology generated by the state space $\mathcal{X}$ allows for point evaluation of the $v$ component, but it does not allow us to define a pointwise evaluation of $v_{t}$-roughly speaking, $1 / 2+\varepsilon$ derivatives are missing. In order to handle this difficulty in the case of $\mathcal{C}_{2}$, we shall use "additional" smoothness results developed for structural acoustic problems in [2], which say that $v_{t}$ has greater smoothness than the state space guarantees; in particular, $v_{t} \in L^{2}\left(0, T ; H^{2}\left(\Gamma_{0}\right)\right)$. This allows us to use Sobolev's imbeddings to obtain the well-posedness with the $\mathcal{C}_{2}$ observation.

In the case of the observation operator $\mathcal{C}_{3}$, the situation is much more delicate. The state space guarantees $L^{2}$ smoothness of $z_{t}$ in the cavity. However, in order to use a Sobolev's imbedding one would need to have $z_{t} \in H^{3 / 2+\varepsilon}(\Omega)$, so $3 / 2+\varepsilon$ derivatives are missing. It can be shown (even for the one-dimensional example) that $L^{2}\left(0, T ; H^{2}\left(\Gamma_{0}\right)\right)$ tangential smoothness of the boundary input $v_{t}$ does not produce sufficient interior smoothness for the variable $z_{t}$ to make Sobolev embeddings useful for interior-point evaluation. In fact, one could show (with additional nontrivial work) that the maximal internal smoothness of $z_{t}$ is $H^{1 / 2}(\Omega)$, but even this does not suffice to take pointwise evaluation in two dimensions. To cope with this difficulty, we resort to completely different arguments, which are based on microlocal analysis for the general smooth domains, and on very delicate calculations involving harmonic analysis when $\Omega$ is a rectangle. The rectangular case is dealt with in section 3 , while the smooth domain case is treated in [5].

Proposition 2.3. The system (2.2) with observation $y(t)=\mathcal{C}_{1} X(t)$ or observation $y(t)=\mathcal{C}_{2} X(t)$ is well posed.

Proof. Condition (1) in Definition 2.1 has already been established in [2]. Condition (2) follows immediately from Lemma 2.1 in [2]. To verify condition (3), first 
note that $\mathcal{C}_{1}$ is bounded on $\mathcal{X}$, hence is an admissible observation operator for any semigroup on $\mathcal{X}$. Now let the projections $P_{0}$ and $P_{1}$ be defined by

$$
\begin{aligned}
& P_{0}\left[z_{1}, z_{2}, v_{1}, v_{2}\right]^{T}=\left[v_{1}, v_{2}\right]^{T}, \\
& P_{1}\left[z_{1}, z_{2}, v_{1}, v_{2}\right]^{T}=\left[z_{1}, z_{2}\right]^{T} .
\end{aligned}
$$

Theorem 1.1 in [2] shows that for any $X_{0} \in \mathcal{X}$ and $T>0$,

$$
P_{0} S(\cdot) X_{0} \in L^{2}\left(0, T ; \mathcal{D}\left(\AA^{\frac{1}{2}}\right) \times \mathcal{D}\left(\AA^{\frac{1}{2}}\right)\right) .
$$

Let $X_{0} \in \mathcal{X}$ and $X(t)=S(t) X_{0}$ be of the form (2.8). Using the Sobolev embedding theorem and the fact that $\mathcal{D}\left(\AA^{1 / 2}\right)=H_{0}^{2}\left(\Gamma_{0}\right)$, we see that $\mathcal{C}_{2} S(\cdot) X_{0}=v_{t}\left(\cdot, \xi_{0}\right) \in$ $L^{2}(0, T)$. By the principle of uniform boundedness, $\mathcal{C}_{2}$ is an admissible observation operator for $S(t)$.

To verify condition (4), note that Proposition 2.3 in [2] shows that for any $T>0$ and $u \in L^{2}[0, T ; U]$,

$$
P_{0} \int_{0}^{\cdot} S(\cdot-\tau) \mathcal{B} u(\tau) d \tau \in L^{2}\left(0, T ; \mathcal{D}\left(\AA^{\frac{1}{2}}\right) \times \mathcal{D}\left(\AA^{\frac{1}{2}}\right)\right) .
$$

Using the same reasoning as we used for the admissibility of $\mathcal{C}_{1}$ and $\mathcal{C}_{2}$, we see that (2.2) with this observation is well posed.

If $Z$ is a Banach space, define $H_{\alpha}^{\infty}(Z)$ to be all analytic $Z$-valued functions $\mathbf{H}(s)$ for which there exists $M>0$ such that $\|\mathbf{H}(s)\|<M$ for all $s \in \mathbb{C}_{\alpha}$. If $\alpha=0$ and if $Z$ is clear from the context, we denote $H_{\alpha}^{\infty}(Z)$ by $H^{\infty}$. Let $y(t)$ be the observation of a system resulting from a given $u$ and zero initial conditions. If we denote the Laplace transform of $y$ by $\hat{y}$ and if

$$
\hat{y}(s)=\mathbf{H}(s) \hat{u}(s),
$$

then we say that $\mathbf{H}(s)$ is the transfer function of the system. From Proposition 3.2 in [25], (2.19) is equivalent to $\mathbf{H} \in H_{\beta}^{\infty}(\mathcal{L}(U, Y))$, leading to the following definition.

Definition 2.4. A transfer function $\mathbf{H}(s)$ is well posed if $\mathbf{H} \in H_{\beta}^{\infty}(\mathcal{L}(U, Y))$ for some $\beta \in \mathbb{R}$.

Definition 2.5. A transfer function $\mathbf{H}$ (or, equivalently, an input-output map $\mathcal{T})$ is input-output stable if

$\mathbf{H} \in H^{\infty}(\mathcal{L}(U, Y))$ (or, equivalently, if $\left.\mathcal{T} \in \mathcal{L}\left(L^{2}[0, \infty ; U), L^{2}[0, \infty ; Y)\right)\right)$.

Definition 2.6. A system is regular if it is well posed and if the following condition is satisfied by its transfer function $\mathbf{H}(s)$ :

(5) $\lim _{s \rightarrow \infty, s \in \mathbb{R}} \mathbf{H}(s)=: D \in \mathcal{L}(U, Y)$ exists.

In this case we say that $D$ is the feedthrough of the transfer function and of the system.

DEFINITION 2.7. If an input-output system is well posed and the transfer function satisfies condition (5), then we say that the input-output system is regular and that its transfer function is regular.

It is shown in [25] that if a controlled, observed system with semigroup generator $\mathcal{A}$, input operator $\mathcal{B}$, and observation operator $\mathcal{C}$ is regular, it has the following state space representation, with state space $\mathcal{X}$ :

$$
\begin{aligned}
& \dot{X}(t)=\mathcal{A} X(t)+\mathcal{B} u(t), \\
& y(t)=\mathcal{C}_{\Lambda} X(t)+D u(t),
\end{aligned}
$$


where $\mathcal{C}_{\Lambda}$ is the Lebesgue extension of $\mathcal{C}$; see [25].

Remark 2.8. If a controlled, observed system is well posed but not regular, it has a state space representation, but the abstract form of the observation (when the control is present) is much more complicated (and less analogous to finite dimensional systems) than that in (2.20) (see Salamon [24]).

Since $\mathcal{B}$ is an admissible input operator and $\mathcal{C}_{1}$ is a bounded observation operator, we get the following simple result, which follows immediately from [25].

Proposition 2.9. The system (2.2) with observation $\mathcal{C}_{1} X(t)$ is regular.

3. Observation of acoustic pressure. In the case where the observation operator is $\mathcal{C}_{3}$ (given in (2.17)) the well-posedness of the system is a much more delicate issue than the case treated by Proposition 2.3. This observation is not an admissible observation for $S(t)$ when the state space is $\mathcal{X}$. However, we will see in Corollary 3.4 that when $\Omega$ is a rectangular region, the input-output system is well posed. In Theorem 4.8 we see that the transfer function for $(2.2)$ with this observation is regular, i.e., it also satisfies condition (5) in Definition 2.6.

Let $T>0$ and let $\Omega$ be a rectangular domain in $\mathbb{R}^{2}$ with boundary $\Gamma$. Let $\left[z(t), z_{t}(t)\right]^{T}$ be the solution of the wave equation

$$
\begin{aligned}
& z_{t t}(t, x, y)=\Delta z(t, x, y) \quad \text { on }(0, T) \times \Omega, \\
& \frac{\partial z}{\partial \nu}(t, \zeta)=\mu(t, \zeta) \quad \text { on }(0, T) \times \Gamma, \\
& z(0, x, y)=z_{t}(0, x, y)=0 \quad \text { on } \Omega .
\end{aligned}
$$

With boundary data $\mu \in L^{2}\left(0, T ; H^{5 / 4}(\Gamma)\right)$, we already know from Avalos [4] that

$$
\left[z, z_{t}\right] \in C\left([0, T] ; H^{1}(\Omega) \times L^{2}(\Omega)\right) .
$$

We assume that $\mu(t, \cdot)=0$ for $t>T$. We now prove the following "trace" result, which allows for pointwise evaluation of the velocity $z_{t}$ at a point in $\bar{\Omega}$.

TheOREM 3.1. For every fixed $\left(x_{0}, y_{0}\right) \in \bar{\Omega}$, the mapping $\mu \rightarrow z_{t}\left(\cdot, x_{0}, y_{0}\right)$ is in

$$
\mathcal{L}\left(L^{2}\left(0, T ; H^{5 / 4}(\Gamma)\right), L^{2}(0, T)\right) .
$$

In particular, there exists $M>0$, independent of $\left(x_{0}, y_{0}\right)$, such that for every $\mu \in$ $L^{2}\left(0, T ; H^{5 / 4}(\Gamma)\right)$,

$$
\left\|z_{t}\left(\cdot, x_{0}, y_{0}\right)\right\|_{L^{2}(0, T)} \leq M\|\mu\|_{L^{2}\left(0, T ; H^{\frac{5}{4}}(\Gamma)\right)} \cdot
$$

Remark 3.2. Note that this result does not follow from a direct application of the classical Sobolev embedding theorem.

Proof. Without loss of generality, we can set $\Omega:=\left\{(x, y) \in \mathbb{R}^{2} \mid 0<x, y<\pi\right\}$, and since the mapping of concern here is linear, we can set $u \equiv 0$ except on the side $\{y=0,0 \leq x \leq \pi\}$. Fix $\left(x_{0}, y_{0}\right) \in \bar{\Omega}$. Let $\left\{\lambda_{m n}, \Phi_{m n}\right\}_{m, n=1}^{\infty}$ denote, respectively, the eigenvalues and orthonormalized eigenfunctions of the operator $A$ defined in section 2. These are given explicitly by

$$
\begin{aligned}
& \lambda_{m n}=n^{2}+m^{2} \quad \text { for } m, n=1,2, \ldots, \\
& \Phi_{m n}(x, y)=\frac{2}{\pi} \cos n x \cos m y \text { for } m, n=1,2, \ldots .
\end{aligned}
$$


We can explicitly write out the solution $\left[z, z_{t}\right]$ of $(3.1)$ — this is done in [4] and [16] —as

$$
\begin{aligned}
& z(t, x, y)=\sum_{m, n=1}^{\infty}\left\{\frac{1}{\sqrt{n^{2}+m^{2}}} \int_{0}^{t} \sin \sqrt{n^{2}+m^{2}}(t-\tau) \mu_{n}(\tau) d \tau\right\} \Phi_{m n}(x, y) \\
& z_{t}(t, x, y)=\sum_{m, n=1}^{\infty}\left\{\int_{0}^{t} \cos \sqrt{n^{2}+m^{2}}(t-\tau) \mu_{n}(\tau) d \tau\right\} \Phi_{m n}(x, y)
\end{aligned}
$$

where

$$
\mu_{n}(t):=\frac{2}{\pi} \int_{0}^{\pi} \mu(t, \xi) \cos (n \xi) d \xi
$$

We now need the following proposition.

Proposition 3.3. For arbitrary $y_{0} \in[0, \pi]$, the map $\mu \rightarrow z_{t}\left(\cdot, \cdot, y=y_{0}\right)$ is in

$$
\mathcal{L}\left(L^{2}\left(0, T ; H^{\frac{5}{4}}(0, \pi)\right), L^{2}\left(0, T ; H^{\frac{3}{4}}(0, \pi)\right)\right) .
$$

In particular, there exists $M>0$, independent of $y_{0} \in[0, \pi]$, such that for all $\mu \in$ $L^{2}\left(0, T ; H^{\frac{5}{4}}(0, \pi)\right)$,

$$
\left\|z_{t}\left(\cdot, \cdot, y=y_{0}\right)\right\|_{L^{2}\left(0, T ; H^{\frac{3}{4}}(0, \pi)\right)} \leq M\|\mu\|_{L^{2}\left(0, T ; H^{\frac{5}{4}}(0, \pi)\right)} .
$$

Proof of Proposition 3.3. Define the operator $A_{\Pi}: L^{2}(0, \pi) \rightarrow L^{2}(0, \pi)$ by

$$
A_{\Pi}=-\frac{d^{2}}{d x^{2}} \quad \text { with } \mathcal{D}\left(A_{\Pi}\right)=\left\{g \in H^{2}(0, \pi) \mid \frac{\partial g(\pi)}{\partial x}=\frac{\partial g(0)}{\partial x}=0\right\} .
$$

$A_{\Pi}$ is self-adjoint, positive semidefinite, so its positive fractional powers are well defined. Its respective eigenvalues and orthonormalized eigenvectors $\left\{\lambda_{n}, \Phi_{n}\right\}_{n=0}^{\infty}$ are given explicitly by

$$
\lambda_{n}=n^{2} \quad \text { and } \Phi_{n}(x)=\frac{2}{\pi} \cos n x .
$$

Then, for $g$ in $L^{2}(0, \pi)$ and $\eta \geq 0$,

$$
A_{\Pi}^{\frac{\eta}{2}} g=A_{\Pi}^{\frac{\eta}{2}} \sum_{n=1}^{\infty}\left(g, \Phi_{n}\right) \Phi_{n}=\sum_{n=1}^{\infty}\left(g, \Phi_{n}\right) n^{\eta} \Phi_{n}
$$

with the $L^{2}$-convergence of the series on the right-hand side occurring if and only if $g \in D\left(A_{\Pi}^{\frac{\eta}{2}}\right)$. Using this equality and the explicit representation of $z_{t}$ in (3.3), we obtain

$$
A_{\Pi}^{\frac{\eta}{2}} z_{t}\left(t, \cdot, y=y_{0}\right)=\sum_{m, n=1}^{\infty} n^{\eta}\left\{\int_{0}^{t} \cos \sqrt{n^{2}+m^{2}}(t-\tau) \mu_{n}(\tau) d \tau\right\} \Phi_{n}(\cdot) \cos m y_{0} .
$$

Furthermore, from [14], for all $g \in H^{\eta}(0, \pi), 0 \leq \eta<3 / 2$, we can take

$$
\|g\|_{H^{\eta}(0, \pi)}^{2}=\left\|A_{\Pi}^{\frac{\eta}{2}} g\right\|_{L^{2}(0, \pi)}^{2} .
$$


Using this and the orthonormality of $\left\{\Phi_{n}\right\}$, we have that for every $g \in H^{\eta}(0, \pi)$, $0 \leq \eta \leq 3 / 2$,

$$
\|g\|_{H^{\eta}(0, \pi)}^{2}=\sum_{n=1}^{\infty}\left|\left(g, \Phi_{n}\right)\right|^{2} n^{2 \eta} .
$$

Extending the boundary input $\mu$ by zero outside the interval $[0, T]$, we take the Laplace transform in time of both sides of (3.5) with transform variable

$$
\lambda=\gamma+i \omega
$$

to thereby obtain

$$
\widehat{A_{\Pi}^{\frac{\eta}{2}}} z_{t}\left(\lambda, \cdot, y=y_{0}\right)=\sum_{m, n=1}^{\infty} \frac{\lambda n^{\eta}}{\lambda^{2}+\lambda_{m n}} \widehat{\mu_{n}}(\lambda) \Phi_{n}(\cdot) \cos m y_{0}
$$

Therefore, we can use the generalized Parseval's relation (see [13, p. 212]), (3.6), (3.8), and (3.7) to obtain for fixed $\eta \in[0,3 / 2]$ and any $\gamma \geq 0$

$$
\begin{aligned}
& 2 \pi \int_{0}^{\infty} e^{-2 \gamma t}\left\|z_{t}\left(t, \cdot, y=y_{0}\right)\right\|_{H^{\eta}(0, \pi)}^{2} d t=2 \pi \int_{0}^{\infty} e^{-2 \gamma t}\left\|A_{\Pi}^{\frac{\eta}{2}} z_{t}\left(t, \cdot, y=y_{0}\right)\right\|_{L^{2}(0, \pi)}^{2} d t \\
= & \int_{-\infty}^{\infty}\left\|\widehat{A_{\Pi}^{\frac{\eta}{2}}} z_{t}\left(\gamma+i \omega, \cdot, y=y_{0}\right)\right\|_{L^{2}(0, \pi)}^{2} d \omega \\
= & \int_{-\infty}^{\infty} \sum_{n=1}^{\infty}\left|\sum_{m=1}^{\infty} \frac{\lambda}{\lambda^{2}+\lambda_{m n}} \cos m y_{0}\right|^{2}\left|\widehat{\mu_{n}}(\lambda)\right|^{2} n^{2 \eta} d \omega .
\end{aligned}
$$

We now fix $\gamma>0$ and specify that $\eta \in[0,5 / 4]$. Suppose we can find some $\theta \in$ $[0,5 / 4-\eta]$ and some positive constant $C_{0}>0$ such that

$$
\frac{1}{n^{\theta}}\left|\sum_{m=1}^{\infty} \frac{\lambda}{\lambda^{2}+\lambda_{m n}} \cos m y_{0}\right|<C_{0},
$$

where $C_{0}$ does not depend on $\omega \in \mathbb{R}$ or $n=1,2, \ldots$. Then (3.9) is equal to

$$
\begin{gathered}
\int_{-\infty}^{\infty} \sum_{n=1}^{\infty}\left|\sum_{m=1}^{\infty} \frac{\lambda n^{-\theta}}{\lambda^{2}+\lambda_{m n}} \cos m y_{0}\right|^{2}\left|\widehat{\mu_{n}}(\lambda)\right|^{2} n^{2(\eta+\theta)} d \omega \\
\leq C_{0} \int_{-\infty}^{\infty} \sum_{n=1}^{\infty}\left|\widehat{\mu_{n}}(\lambda)\right|^{2} n^{2(\eta+\theta)} d \omega=C_{0} \int_{-\infty}^{\infty}\left\|\widehat{A_{\Pi}^{\frac{\eta+\theta}{2}}} \mu(\gamma+i \omega)\right\|_{L^{2}(0, \pi)}^{2} d \omega \\
(3.11)=2 \pi C_{0} \int_{0}^{T} e^{-2 \gamma t}\|\mu(t)\|_{H^{\eta+\theta}(0, \pi)}^{2} d t \leq 2 \pi C_{0} \int_{0}^{T}\|\mu(t)\|_{H^{\frac{5}{4}}(0, \pi)}^{2} d t .
\end{gathered}
$$

Hence, if we can verify (3.10) for some $\theta \in[0,5 / 4-\eta]$, then (3.9) and (3.11) imply that there exists $C_{1}$ such that

$$
\int_{0}^{T}\left\|z_{t}\left(t, \cdot, y=y_{0}\right)\right\|_{H^{\eta}(0, \pi)}^{2} d t \leq C_{1} \int_{0}^{T}\|\mu(t)\|_{H^{5 / 4}(0, \pi)}^{2} .
$$


To attain the sought-after estimate (3.10), it suffices to ascertain the convergence of each of the following sums for some value of $\theta \in[0,5 / 4-\eta]$, with each convergence being again independent of $\omega \in \mathbb{R}$ and $n \in \mathbb{N}$ :

(i) $\frac{1}{n^{\theta}}\left|\sum_{m=1}^{\infty} \frac{2 \gamma \omega^{2}}{\left(\gamma^{2}-\omega^{2}+m^{2}+n^{2}\right)^{2}+4 \gamma^{2} \omega^{2}}\right|$,
(ii) $\frac{1}{n^{\theta}}\left|\sum_{m=1}^{\infty} \frac{\omega\left(\gamma^{2}-\omega^{2}+m^{2}+n^{2}\right)}{\left(\gamma^{2}-\omega^{2}+m^{2}+n^{2}\right)^{2}+4 \gamma^{2} \omega^{2}}\right|$.

In fact, the absolute convergence of these sums, independent of $\omega$ and $n$, has already been shown in [4] for $\theta=1 / 2$ (see in particular equations (3.19), (3.33), (3.55), and (3.76) in [4]). With the estimate (3.10) being established for $\theta=1 / 2$, we see that (3.12) is true for any $\eta$ such that $\theta=1 / 2 \in[0,5 / 4-\eta]$ and so in particular for $\eta=3 / 4$.

Conclusion of the proof of Theorem 3.1. Now upon the use of the Sobolev embedding theorem in one dimension and Proposition 3.3, we have that for any $\left(x_{0}, y_{0}\right) \in[0, \pi] \times[0, \pi]$, there exists $M, \tilde{M}>0$ such that

$$
\begin{aligned}
\int_{0}^{T}\left|z_{t}\left(t, x=x_{0}, y=y_{0}\right)\right|^{2} d t & \leq \tilde{M} \int_{0}^{T}\left\|z_{t}\left(t, \cdot, y=y_{0}\right)\right\|_{H^{\frac{3}{4}}(0, \pi)}^{2} d t \\
& \leq M\|\mu\|_{L^{2}\left(0, T ; H^{\frac{5}{4}}(0, \pi)\right)}^{2}
\end{aligned}
$$

This finishes the proof of Theorem 3.1.

COROLLARY 3.4. The system (2.2) with observation $y(t)=\mathcal{C}_{3} X(t)$ is input-output well posed.

Proof. The first three equations in (2.2) are of the same form as the wave equation (3.1) when $\mu=0$ on $\Gamma \backslash \Gamma_{0}$ and $\mu=v_{t}$ on $\Gamma_{0}$. Theorem 1.1 in [2] implies that there exists $M_{1}>0$ such that

$$
\left\|v_{t}\right\|_{L^{2}\left(0, T ; H^{2}\left(\Gamma_{0}\right)\right)} \leq M_{1}\|u\|_{L^{2}(0, T ; U)} .
$$

Combining this with $(3.2)$ and the fact that $\|\cdot\|_{H^{5 / 4}\left(\Gamma_{0}\right)} \leq\|\cdot\|_{H^{2}\left(\Gamma_{0}\right)}$, we see that there exists $M_{2}>0$ such that

$$
\left\|z_{t}\left(\cdot, x_{0}, y_{0}\right)\right\|_{L^{2}(0, T)} \leq M_{2}\|u\|_{L^{2}(0, T ; U)},
$$

so the input-output map from $u \in L^{2}(0, T ; U)$ into $\mathcal{C}_{3} X=z_{t}\left(\cdot, x_{0}, y_{0}\right) \in L^{2}(0, T)$ is well posed.

4. Regularity results. In this section we show that when the observation is $\mathcal{C}_{2} X(t)$ the system is regular, and when the observation is $\mathcal{C}_{3} X(t)$ the system is inputoutput regular. Since we have shown that the system is well posed in the former case and input-output well posed in the latter case, it suffices to show in both of these cases that the transfer function satisfies condition (5) in Definition 2.6. We first need a few technical lemmas.

Let $\alpha>0$-we will eventually specialize to $\alpha$ as in (2.1), but all the following lemmas are true for more general $\alpha$. For $s>0$, let $\mathbf{T}(s)$ be defined on $\mathcal{D}\left(\AA^{\alpha / 4}\right)^{\prime}$ by

$$
\mathbf{T}(s):=\left[\frac{s^{2}}{s+1}+\mathbf{A}\right]^{-1} \text {. }
$$


LEMMA 4.1. For

$$
0 \leq \theta<4-\alpha
$$

there exists $M>0$ such that for all $s>0$,

$$
\|\mathbf{T}(s)\|_{\mathcal{L D}\left(\mathbf{A}^{\circ \alpha / 4}\right)^{\prime}, \mathcal{D}\left(\mathbf{A}^{\circ \theta / 4}\right)} \leq \frac{M}{\left(1+\frac{s^{2}}{s+1}\right)^{1-\frac{1}{4}(\theta+\alpha)}} .
$$

Proof. For $\theta$ in the prescribed range and $w \in \mathcal{D}\left(\AA^{\alpha / 4}\right)^{\prime}$, we have

$$
\begin{aligned}
\|\mathbf{T}(s) w\|_{\mathcal{D}\left(\AA^{\theta / 4}\right)} & =\left\|\left[\frac{s^{2}}{s+1}+\AA\right]^{-1} w\right\|_{\mathcal{D}\left(\AA^{\theta / 4}\right)} \\
=\left\|\AA^{\frac{\theta}{4}}\left[\frac{s^{2}}{s+1}+\AA\right]^{-1} w\right\|_{L^{2}\left(\Gamma_{0}\right)} & =\left\|\AA^{\frac{\theta}{4}}\left[\frac{s^{2}}{s+1}+\AA\right]^{-1} \AA^{\frac{\alpha}{4}} \AA^{-\frac{\alpha}{4}} w\right\|_{L^{2}\left(\Gamma_{0}\right)} .
\end{aligned}
$$

Using Krein [15, Eq. (5.15), p. 115] and (2.4), we see that if $s>0$, there exists $M>0$ such that

$$
\begin{aligned}
\|\mathbf{T}(s) w\|_{\mathcal{D}\left(\AA^{\theta / 4}\right)} & \leq \frac{M}{\left[1+\frac{s^{2}}{s+1}\right]^{1-\frac{1}{4}(\theta+\alpha)}}\left\|\AA^{-\frac{\alpha}{4}} w\right\|_{L^{2}\left(\Gamma_{0}\right)} \\
& =\frac{M}{\left[1+\frac{s^{2}}{s+1}\right]^{1-\frac{1}{4}(\theta+\alpha)}}\|w\|_{\mathcal{D}\left(\AA^{\alpha / 4}\right)^{\prime}}
\end{aligned}
$$

Remark 4.2. The same type of estimate could be obtained using the fact that $\AA$ generates an analytic semigroup, but we did not use analyticity here.

LEMMA 4.3. For large enough $s>0$ the operator

$$
I+\frac{s^{2}}{s+1} \mathbf{T}(s) N^{*} A\left(s^{2}+A\right)^{-1} A N
$$

is boundedly invertible on $\mathcal{L}\left(\mathcal{D}\left(\AA^{\circ / 4}\right)\right)$ for $\theta \in[0,4-\alpha)$. In particular, there exists $M>0$ such that for large enough $s>0$,

$$
\left\|\left(I+\frac{s^{2}}{s+1} \mathbf{T}(s) N^{*} A\left(s^{2}+A\right)^{-1} A N\right)^{-1}\right\|_{\mathcal{L}\left(\mathcal{D}\left(\mathbf{A}^{\circ \theta / 4}\right)\right)} \leq M .
$$

Proof. We prove this result by considering the Neumann series for

$$
\left(I+\frac{s^{2}}{s+1} \mathbf{T}(s) N^{*} A\left(s^{2}+A\right)^{-1} A N\right)^{-1}
$$

in particular, we show that

$$
\lim _{s \rightarrow \infty}\left\|\frac{s^{2}}{s+1} \mathbf{T}(s) N^{*} A\left(s^{2}+A\right)^{-1} A N\right\|_{\mathcal{L}\left(\mathcal{D}\left(\AA^{\theta / 4}\right)\right)}=0 .
$$


Let $w \in \mathcal{D}\left(\AA^{\theta / 4}\right)$. From $(2.7), N^{*} A \in \mathcal{L}\left(\mathcal{D}\left(A^{\frac{1}{4}+\epsilon}\right), L^{2}\left(\Gamma_{0}\right)\right)$, where $\epsilon>0$ is arbitrarily small, so there exists $M>0$ such that

$$
\begin{aligned}
\left\|N^{*} A\left(s^{2}+A\right)^{-1} A N w\right\|_{\mathcal{D}\left(\AA^{\alpha / 4}\right)^{\prime}} \leq\left\|N^{*} A\left(s^{2}+A\right)^{-1} A N w\right\|_{L^{2}\left(\Gamma_{0}\right)} \\
\leq M\left\|\left(s^{2}+A\right)^{-1} A N w\right\|_{D\left(A^{\frac{1}{4}+\epsilon}\right)} \\
=M\left\|A^{\frac{1}{4}+\epsilon}\left(s^{2}+A\right)^{-1} A N w\right\|_{L^{2}(\Omega)} \\
=M\left\|A^{\frac{1}{2}+2 \epsilon}\left(s^{2}+A\right)^{-1} A^{\frac{3}{4}-\epsilon} N w\right\|_{L^{2}(\Omega)} .
\end{aligned}
$$

Using equation (5.15) on page 115 in [15] and (2.7), we see that

$$
(4.5) \leq \frac{M}{\left(1+s^{2}\right)^{\frac{1}{2}-2 \epsilon}}\left\|A^{\frac{3}{4}-\epsilon} N w\right\|_{L^{2}(\Omega)} \leq \frac{M}{\left(1+s^{2}\right)^{\frac{1}{2}-2 \epsilon}}\|w\|_{L^{2}\left(\Gamma_{0}\right)}
$$

and therefore

$$
\left\|N^{*} A\left(s^{2}+A\right)^{-1} A N w\right\|_{\mathcal{D}\left(\AA^{\alpha / 4}\right)^{\prime}} \leq \frac{M}{\left(1+s^{2}\right)^{\frac{1}{2}-2 \epsilon}}\|w\|_{L^{2}\left(\Gamma_{0}\right)} .
$$

Since $\theta>0$, using (4.6) with (4.2) yields for large $s>0$

$$
\frac{s^{2}}{s+1}\left\|\mathbf{T}(s) N^{*} A\left(s^{2}+A\right)^{-1} A N w\right\|_{\mathcal{L}\left(\mathcal{D}\left(\AA^{\theta / 4}\right)\right)}=\mathcal{O}\left(\frac{1}{s^{1-\frac{1}{4}(\theta+\alpha)-4 \epsilon}}\right) .
$$

Since $\theta+\alpha<4$ by hypothesis, for $\epsilon>0$ small enough the equation above implies that (4.4) is true. Hence

$$
\begin{aligned}
& \left\|\left(I+\frac{s^{2}}{s+1} \mathbf{T}(s) N^{*} A\left(s^{2}+A\right)^{-1} A N\right)^{-1}\right\|_{\mathcal{L}\left(\mathcal{D}\left(\AA^{\theta / 4}\right)\right)} \\
& \leq \frac{1}{1-\frac{s^{2}}{s+1}\left\|\mathbf{T}(s) N^{*} A\left(s^{2}+A\right)^{-1} A N w\right\|_{\mathcal{L}\left(\mathcal{D}\left(\AA^{\theta / 4}\right)\right)}}
\end{aligned}
$$

for $s>0$ large enough, which finishes the proof of Lemma 4.3.

For $s>0$, let

$$
\mathbf{H}^{1}(s):=\left\{I+\frac{s^{2}}{s+1} \mathbf{T}(s) N^{*} A\left(s^{2}+A\right)^{-1} A N\right\}^{-1} \frac{s}{s+1} \mathbf{T}(s) B .
$$

Now let $\alpha$ be as in (2.1), and recall that $B \in \mathcal{L}\left(U, H^{-\alpha}\left(\Gamma_{0}\right)\right)$. Since $\mathcal{D}\left(\AA^{\eta / 4}\right)=$ $H^{\eta}{ }_{0}\left(\Gamma_{0}\right)$ for $\eta \in[0,5 / 2)$, we see that $B \in \mathcal{L}\left(U, \mathcal{D}\left(\AA^{\alpha / 4}\right)^{\prime}\right)$. Combining Lemmas 4.1 and 4.3 we immediately obtain the following result for the operator $\mathbf{H}^{1}(s)$.

Lemma 4.4. $\mathbf{H}^{1}(s)$ is an element of $\mathcal{L}\left(U, H^{\theta}\left(\Gamma_{0}\right)\right)$ for $s>0$ large enough and $\theta \in[0,4-\alpha)$ and for every $\theta$ there exists $M>0$ such that

$$
\left\|\mathbf{H}^{1}(s)\right\|_{\mathcal{L}\left(U, \mathcal{D}\left(\mathbf{A}^{\circ \theta / 4}\right)\right)} \leq \frac{M}{\left[1+\frac{s^{2}}{s+1}\right]^{1-\frac{1}{4}(\theta+\alpha)}} .
$$


We now use these lemmas to prove the regularity of the system (2.2) with observation $\mathcal{C}_{2} X(t)$. We first need to show that $\mathbf{H}^{1}$ is the transfer function from $u$ to $v_{t}$.

LEMMA 4.5. Let the solution of (2.2) be of the form (2.8) with $u \in L_{\gamma}^{2}(0, \infty ; U)$ for some $\gamma \in \mathbb{R}$ and with $X(0)=0$. Then, for sufficiently large $s>0$,

$$
\widehat{v_{t}}(s)=\mathbf{H}^{1}(s) \widehat{u}(s) .
$$

Proof. Applying the Laplace transform (in time $t$ ) to $(2.15),(2.16)$ for $s \in \mathbb{C}_{\beta}$, we have, formally,

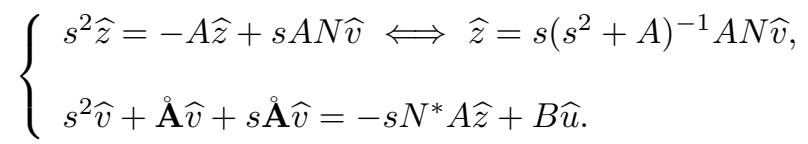

Substituting the first equation above into the second then yields

$$
\left(s^{2}+(s+1) \AA+s^{2} N^{*} A\left(s^{2}+A\right)^{-1} A N\right) \widehat{v}=B \widehat{u} .
$$

To deal with this equation, we will use the relation

$$
\left(s^{2}+(\tilde{A}+\tilde{B})\right)^{-1}=\left[I+\left(s^{2}+\tilde{A}\right)^{-1} \tilde{B}\right]^{-1}\left(s^{2}+\tilde{A}\right)^{-1}
$$

for any operators $\tilde{A}$ and $\tilde{B}$ and scalars $s$ for which all the inverses in the relation exist. Letting $\tilde{A}=(s+1) \AA$ and $\tilde{B}=s^{2} N^{*} A\left(s^{2}+A\right)^{-1} A N$, we obtain formally

$$
\begin{aligned}
& \left(s^{2}+(s+1) \AA+s^{2} N^{*} A\left(s^{2}+A\right)^{-1} A N\right)^{-1} \\
= & \left(I+\left(s^{2}+(s+1) \AA\right)^{-1} s^{2} N^{*} A\left(s^{2}+A\right)^{-1} A N\right)^{-1}\left(s^{2}+(s+1) \AA\right)^{-1} \\
(4.10)= & \left(I+\frac{s^{2}}{s+1} \mathbf{T}(s) N^{*} A\left(s^{2}+A\right)^{-1} A N\right)^{-1} \frac{1}{s+1} \mathbf{T}(s) .
\end{aligned}
$$

Using Lemma 4.3, we see that (4.10) is indeed valid for sufficiently large real $s$. Using (4.10) in (4.9), we get

$$
\widehat{v}(s)=\left(I+\frac{s^{2}}{s+1} \mathbf{T}(s) N^{*} A\left(s^{2}+A\right)^{-1} A N\right)^{-1} \frac{1}{s+1} \mathbf{T}(s) B \widehat{u}(s)
$$

or

$$
\widehat{v_{t}}(s)=s \hat{v}(s)=\mathbf{H}^{1}(s) \widehat{u}(s)
$$

which finishes the lemma.

It follows from Lemma 4.5 that for sufficiently large real $s$ the transfer function from $u(t)$ to $y=\mathcal{C}_{2} X(t)$ is

$$
\mathbf{H}_{1}(s):=C_{\xi_{0}} \mathbf{H}^{1}(s)
$$

(where we recall that $C_{\xi_{0}}$ is the point evaluation at $\xi_{0} \in \Gamma_{0}$ ). Since the system (2.2) with this observation is well posed, there exists $\beta \in \mathbb{R}$ such that $\mathbf{H}_{1} \in H_{\beta}^{\infty}(\mathcal{L}(U, Y))$; hence this transfer function can be extended analytically for $s \in \mathbb{C}_{\beta}$. 
THEOREM 4.6. $\mathbf{H}_{1}(s)$ is a regular transfer function with feedthrough 0.

Proof. The theorem is proved if we can show that

$$
\lim _{s \rightarrow \infty, s \in \mathbb{R}}\left\|C_{\xi_{0}} \mathbf{H}^{1}(s)\right\|_{\mathcal{L}(U, \mathbb{R})}=0 .
$$

For $1 / 2<\theta<4-\alpha$, Lemma 4.4 and the Sobolev embedding theorem imply that there exists $M>0$ such that

$$
\left\|C_{\xi_{0}} \mathbf{H}^{1}(s)\right\|_{\mathcal{L}(U, \mathbb{R})} \leq M\left\|\mathbf{H}^{1}(s)\right\|_{\mathcal{L}\left(U, H^{\theta}\left(\Gamma_{0}\right)\right)} \leq \frac{M}{\left[1+\frac{s^{2}}{s+1}\right]^{1-\frac{1}{4}(\theta+\alpha)}} .
$$

Taking the limit as $s \rightarrow \infty$ along the real axis finishes the proof of Theorem 4.6.

We now turn to the transfer function $\mathbf{H}_{2}(s)$ for $(2.2)$ with the observation $\mathcal{C}_{3} X(t)$. In the previous section it is proved that this transfer function is well posed, so there exists $\beta \in \mathbb{R}$ such that $\mathbf{H}_{2} \in H_{\beta}^{\infty}(\mathcal{L}(U, Y))$. We will show that this transfer function is also regular with feedthrough 0 . Let

$$
\mathbf{H}^{2}(s)=s\left(s^{2}+A\right)^{-1} A N \mathbf{H}^{1}(s) .
$$

We first show that $\mathbf{H}^{2}$ is the transfer function from $u$ into the velocity $z_{t}$ of the wave component.

LEMma 4.7. Let the solution of (2.2) be of the form (2.8) with $u \in L_{\beta}^{2}(0, \infty ; U)$ and $X(0)=0$. Then, for sufficiently large real $s$,

$$
\widehat{z_{t}}(s)=\mathbf{H}^{2}(s) \widehat{u}(s) .
$$

Proof. Applying the Laplace transform in time to (2.15), we obtain

$$
s^{2} \widehat{z}(s)=-A \widehat{z} s+A N \widehat{v_{t}}(s)
$$

or, equivalently, using (4.8),

$$
\widehat{z}=\left(s^{2}+A\right)^{-1} A N \widehat{v}_{t}=\left(s^{2}+A\right)^{-1} A N \mathbf{H}^{1}(s) \widehat{u}(s) ;
$$

(4.12) follows immediately from this.

It follows from Lemma 4.7 that for sufficiently large real $s$ the transfer function from $u(t)$ to $y(t)=\mathcal{C}_{3} X(t)$ is

$$
\mathbf{H}_{2}(s):=C_{x_{0}} \mathbf{H}^{2}(s) .
$$

THEOREM 4.8. $\mathbf{H}_{2}(s)$ is a regular transfer function with feedthrough 0.

Proof. Since $\alpha / 8<1 / 4$, we can choose $\varepsilon \in(0,1 / 4-\alpha / 8)$. Using the Sobolev embedding theorem and (2.3), there exist $M_{1}, M_{2}>0$ such that for $s>0$,

$$
\begin{aligned}
\left\|\mathbf{H}_{2}(s)\right\|_{\mathcal{L}(U, \mathbb{R})} & =\left\|C_{x_{0}} \mathbf{H}^{2}(s)\right\|_{\mathcal{L}(U, \mathbb{R})} \leq M_{1}\left\|\mathbf{H}^{2}(s)\right\|_{\mathcal{L}\left(U, H^{1+\varepsilon}(\Omega) \cap L^{2}(\Omega) / \mathbb{R}\right)} \\
& =M_{2}\left\|\mathbf{H}^{2}(s)\right\|_{\mathcal{L}\left(U, \mathcal{D}\left(A^{(1+\varepsilon) / 2}\right)\right)} .
\end{aligned}
$$

The right side of (4.13) is equal to

$$
\begin{aligned}
& M_{2} s\left\|A^{\frac{1}{2}+\frac{\varepsilon}{2}}\left(s^{2}+A\right)^{-1} A N \mathbf{H}^{1}(s)\right\|_{\mathcal{L}\left(U, L^{2}(\Omega)\right)} \\
& \quad=M_{2} s\left\|A^{\frac{3}{4}+\varepsilon}\left(s^{2}+A\right)^{-1} A^{\frac{3}{4}-\frac{\varepsilon}{2}} N \mathbf{H}^{1}(s)\right\|_{\mathcal{L}\left(U, L^{2}(\Omega)\right)} \\
& \leq \frac{s M_{2}}{\left(1+s^{2}\right)^{\frac{1}{4}-\varepsilon}}\left\|A^{\frac{3}{4}-\frac{\varepsilon}{2}} N \mathbf{H}^{1}(s)\right\|_{\mathcal{L}\left(U, L^{2}(\Omega)\right)} \leq \frac{s M_{3}}{\left(1+s^{2}\right)^{\frac{1}{4}-\varepsilon}}\left\|\mathbf{H}^{1}(s)\right\|_{\mathcal{L}\left(U, L^{2}\left(\Gamma_{0}\right)\right)}
\end{aligned}
$$


for some $M_{3}>0$, where we use equation (5.15) in [15] for the second-to-last inequality and (2.7) for the last inequality. Using Lemma 4.4 with $\theta=0$, the right side of (4.14) is

$$
\leq \frac{s M_{3}}{\left(1+s^{2}\right)^{\frac{1}{4}-\varepsilon}\left(1+\frac{s^{2}}{s+1}\right)^{1-\frac{\alpha}{4}}} .
$$

This shows that

$$
\left\|\mathbf{H}_{2}(s)\right\|_{\mathcal{L}(U, \mathbb{R})}=\mathcal{O}\left(s^{-\frac{1}{2}+2 \varepsilon+\frac{\alpha}{4}}\right) .
$$

By our choice of $\varepsilon$, we see that $-1 / 2+2 \varepsilon+\alpha / 4<0$, so

$$
\lim _{s \rightarrow \infty, s \in \mathbb{R}}\left\|\mathbf{H}_{2}(s)\right\|_{\mathcal{L}(U, \mathbb{R})}=0 .
$$

5. Lack of robustness for dynamic stabilization of regular systems. In this section we present results from the literature on lack of robustness with respect to delays. These results can be easily applied to the structural acoustics system in this paper, or in fact any regular system that satisfies $(2.12)$ and has $R(s, A)$ analytic on $\mathbb{C}_{0}$ and continuous on $\overline{C_{0}}$. We first need to discuss dynamic stabilization in this setting. In the definitions and theorems in this section we deal with generic regular systems, and not just the structural acoustics system in section 2 .

Let $X, X_{c}, U$, and $Y$ be Hilbert spaces, and recall that $X_{-1}$ is defined as in (2.14). Let $\Sigma_{p}$ be a regular system represented by

$$
\begin{gathered}
\dot{x}_{p}=A x_{p}+B u_{p}, \\
y_{p}=C_{\Lambda} x_{p}+D u_{p},
\end{gathered}
$$

where $A$ generates a semigroup on $X, B: U \rightarrow X_{-1}, C: \mathcal{D}(A) \rightarrow Y$, and $D: U \rightarrow Y$, where we recall that $C_{\Lambda}$ is the Lebesgue extension of $C$; see [25]. The subscript $p$ stands for plant, the system we wish to stabilize. The transfer function for (5.1), (5.2) is $\mathbf{H}(s):=C_{\Lambda} R(s, A) B+D$.

Let $\Sigma_{c}$ be a regular system represented by

$$
\begin{aligned}
& \dot{x}_{c}=A^{c} x_{c}+B^{c} u_{c}, \\
& y_{c}=C_{\Lambda}^{c} x_{c}+D^{c} u_{c},
\end{aligned}
$$

where $A^{c}$ generates a semigroup on the Hilbert space $X_{c}, B^{c}: Y \rightarrow\left(X_{c}\right)_{-1}, C^{c}$ : $\mathcal{D}\left(A^{c}\right) \rightarrow U$, and $D^{c}: Y \rightarrow U$. The subscript $c$ stands for controller. The transfer function for (5.3), (5.4) is $\mathbf{H}^{c}(s):=C_{\Lambda}^{c} R\left(s, A^{c}\right) B^{c}+D^{c}$.

We can formally form a closed loop of $\Sigma_{p}$ and $\Sigma_{c}$ by letting

$$
\begin{aligned}
& u_{p}=y_{c}+v_{p}, \\
& u_{c}=y_{p}+v_{c},
\end{aligned}
$$

where we assume that the dimension of $u_{p}$ is equal to the dimension of $y_{c}$ and the dimension of $u_{c}$ is equal to the dimension of $y_{p}$. This closed loop is illustrated in Figure 5.1 (when $\varepsilon=0$ ). 


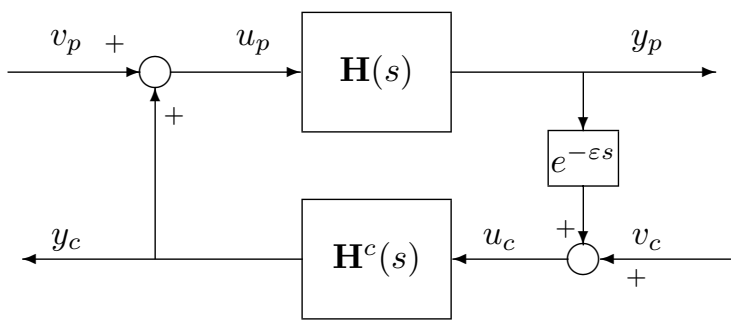

FIG. 5.1. The closed loop of $\Sigma_{p}$ and $\Sigma_{c}$.

There are several difficulties associated with making the connections (5.5) and (5.6); these are covered in detail in Weiss and Curtain [27]. Briefly, we assume that $\mathbf{H}^{c}$ is an admissible feedback transfer function for $\mathbf{H}$, which means that $I-\mathbf{H}^{c}(s) \mathbf{H}(s)$ is invertible for all $s$ in some right half plane, and its inverse is a well posed $\mathcal{L}(U)$ valued transfer function. This condition guarantees that the parallel connection of $\Sigma_{p}$ and $\Sigma_{c}$ is a well-posed linear system $\Sigma_{p, c}$ with state $\left[x_{p}, x_{c}\right]^{T} \in X \times X_{c}$, input $\left[v_{p}, v_{c}\right]^{T} \in U \times Y$, and output $\left[y_{p}, y_{c}\right]^{T} \in Y \times U$. If we assume in addition that $I-D^{c} D$ is invertible in $\mathcal{L}(U)$, then it is shown in [27] that $\Sigma_{p, c}$ is regular as well, with generating operator

$$
A_{p, c}:=\left[\begin{array}{cc}
A+B\left(I-D^{c} D\right)^{-1} D^{c} C_{\Lambda} & B\left(I-D^{c} D\right)^{-1} C_{\Lambda}^{c} \\
B^{c}\left(I-D D^{c}\right)^{-1} C_{\Lambda} & A^{c}+B^{c}\left(I-C C^{c}\right)^{-1} D C_{\Lambda}^{c}
\end{array}\right]
$$

on its natural domain.

DEFINITION 5.1. The regular system $\Sigma_{c}$ is a regular stabilizing controller for $\Sigma_{p}$ if $\mathbf{H}^{c}$ is an admissible feedback transfer function for $\mathbf{H}, I-D^{c} D$ is invertible in $\mathcal{L}(U)$, and $A_{p, c}$ generates an exponentially stable semigroup on $X \times X_{c}$.

Remark 5.2. The above definition of a regular stabilizing controller includes static feedback as a special case, since a regular controller can be of the form $y=$ $D^{c} u$. In this case $A_{p, c}$ generates an exponentially stable semigroup if and only if $A+B\left(I-D^{c} D\right)^{-1} D^{c} C_{\Lambda}$ does.

We now consider the effect of a time delay in the plant output. Let $\varepsilon>0$ and suppose that (5.6) is replaced by

$$
u_{c}(t)=y_{p}(t-\varepsilon)+v_{c}(t),
$$

which is illustrated in Figure 5.1. The transfer function for this system is

$$
\mathbf{F}^{\varepsilon}\left(\mathbf{H}, \mathbf{H}^{c}\right):=\left[\begin{array}{cc}
\mathbf{H}\left(I-e^{-\varepsilon} \cdot \mathbf{H}^{c} \mathbf{H}\right)^{-1} & \mathbf{H H}^{c}\left(I-e^{-\varepsilon \cdot} \mathbf{H} \mathbf{H}^{c}\right)^{-1} \\
e^{-\varepsilon \cdot} \mathbf{H}^{c} \mathbf{H}\left(I-e^{-\varepsilon} \cdot \mathbf{H}^{c} \mathbf{H}\right)^{-1} & \mathbf{H}^{c}\left(I-e^{-\varepsilon \cdot} \mathbf{H} \mathbf{H}^{c}\right)^{-1}
\end{array}\right],
$$

that is,

$$
\left[\hat{v}_{p}, \hat{v}_{c}\right]^{T}=\mathbf{F}^{\varepsilon}\left[\hat{y}_{p}, \hat{y}_{c}\right]^{T} .
$$

Definition 5.3. Suppose $\mathbf{F}^{0}\left(\mathbf{H}, \mathbf{H}^{c}\right)$ is input-output stable (see Definition 2.5). Then we say that the input-output stability of $\mathbf{F}^{0}\left(\mathbf{H}, \mathbf{H}^{c}\right)$ is robust with respect to delays if there exists $\varepsilon^{*}>0$ such that $\mathbf{F}^{\varepsilon}\left(\mathbf{H}, \mathbf{H}^{c}\right)$ is input-output stable for all $\varepsilon \in$ $\left[0, \varepsilon^{*}\right)$. 
Remark 5.4. In the case where the controller is static, it is easy to show that $\mathbf{F}^{\varepsilon}\left(\mathbf{H}, \mathbf{H}^{c}\right)$ is input-output stable if and only if $\left(I-e^{-\varepsilon} \cdot \mathbf{H} \mathbf{H}^{c}\right)^{-1}$ is input-output stable. Hence in this case the input-output stability of $\mathbf{F}^{0}\left(\mathbf{H}, \mathbf{H}^{c}\right)$ is robust with respect to delays if there exists $\varepsilon^{*}>0$ such that $\left(I-e^{-\varepsilon \cdot} \mathbf{H} D^{c}\right)^{-1}$ is input-output stable for all $\varepsilon \in\left[0, \varepsilon^{*}\right)$, and we say that the input-output stability of $(I-\mathbf{H} D)^{-1}$ is robust with respect to delays.

Remark 5.5. If a regular system is exponentially stable (i.e., the semigroup is exponentially stable), then it is input-output stable; see Weiss [25]. Hence, if a regular transfer function is not stable, no regular realization of it is going to be exponentially stable. On the other hand, input-output stability does not necessarily imply exponential stability of the underlying semigroup generator, or even strong stability. Hence, even if we do not identify a state space realization for $\mathbf{F}^{\varepsilon}\left(\mathbf{H}, \mathbf{H}^{c}\right)$, a lack of robustness of the input-output stability of $\mathbf{F}^{0}\left(\mathbf{H}, \mathbf{H}^{c}\right)$ with respect to delays is quite strong.

We now present results about lack of robustness with respect to delays, and apply these results to our structural acoustics model. These results are simple corollaries of the following frequency domain theorem from [17].

TheOrem 5.6 ([17, Thm. 8.5]). Suppose $U$ and $Y$ are finite dimensional, $\mathbf{H H}^{c}$ is regular, and $\mathbf{F}^{0}\left(\mathbf{H}, \mathbf{H}^{c}\right)$ is input-output stable. If

$$
\limsup _{|s| \in \mathbb{C}_{0}, s \rightarrow \infty}\|\mathbf{H}(s)\|_{\mathcal{L}(U, Y)}=\infty,
$$

then the input-output stability of $\mathbf{F}^{0}\left(\mathbf{H}, \mathbf{H}^{c}\right)$ is not robust with respect to delays. In particular, there exist sequences $\left\{\varepsilon_{n}\right\}$ and $\left\{p_{n}\right\}$ with

$$
\varepsilon_{n}>0, \quad \varepsilon_{n} \rightarrow 0, \quad p_{n} \in \mathbb{C}_{0}, \quad\left|\operatorname{Im}\left(p_{n}\right)\right| \rightarrow \infty,
$$

such that for any $n \in \mathbb{N}, p_{n}$ is a pole of $\mathbf{H H}^{c}\left(I-e^{-\varepsilon_{n}} \cdot \mathbf{H} \mathbf{H}^{c}\right)^{-1}$ and hence of the overall closed-loop transfer function $\mathbf{F}^{e}\left(\mathbf{H}, \mathbf{H}^{c}\right)$.

The following two corollaries will be useful for the system under consideration in this paper.

Corollary 5.7. Suppose $U$ and $Y$ are finite dimensional, $\Sigma_{p}=(A, B, C)$ is a regular system, $R(s, A)$ is analytic on $\mathbb{C}_{0}$ and continuous on $\overline{C_{0}}$, and

$$
\limsup _{|s| \rightarrow \infty, s \in \mathbb{C}_{0}}\|R(s, A)\|_{\mathcal{L}(X)}=\infty .
$$

If there exists a regular stabilizing controller $\Sigma_{c}$ for $\Sigma_{p}$, then the input-output stability of $\mathbf{F}^{0}\left(\mathbf{H}, \mathbf{H}^{c}\right)$ is not robust with respect to delays. In particular, the conclusions in Theorem 5.6 hold.

Proof. In Weiss and Rebarber [28] it is shown that if there exists a regular stabilizing controller for $\Sigma_{p}$, then (5.9) holds if and only if (5.10) holds, so we can apply Theorem 5.6.

As a special case of Corollary 5.7, we consider the static feedback case. We do not need the analyticity of $R(s, A)$ in $\mathbb{C}_{0}$ here, since we use the results in [21] instead of the results in [28].

Corollary 5.8. Suppose $U$ and $Y$ are finite dimensional, $\Sigma_{p}=(A, B, C)$ is a regular system, and (5.10) holds. If there exists $K \in \mathcal{L}(Y, U)$ such that the closed-loop generator $A+B K C_{\Lambda}$ generates an exponentially stable semigroup, then the stability of $(I-\mathbf{H} K)^{-1}$ is not robust with respect to delays. In particular, the conclusions in Theorem 5.6 hold for the poles of $\left(I+e^{\varepsilon_{n}} \cdot \mathbf{H} D K\right)^{-1}$. 
We now turn to the structural acoustics model we have analyzed in previous sections. Since this system satisfies the conditions in Corollary 5.7, we can immediately apply Corollaries 5.7 and 5.8.

Case 1. Suppose the observation is given by

$$
\mathcal{C} X(t)=\left[v\left(\alpha_{1}\right), \ldots, v\left(\alpha_{j}\right), v_{t}\left(\beta_{1}\right), \ldots, v_{t}\left(\beta_{k}\right)\right]^{T}
$$

for $\left\{\alpha_{i}\right\}_{i=1}^{j},\left\{\beta_{i}\right\}_{i=1}^{k} \subset \Gamma_{0}$. In particular, we assume in this case that the observation does not include any point evaluations of acoustic pressure. Then, by Proposition 2.3 and Theorem 4.6, the system (2.2) with this observation is a regular system, which can be represented by (2.20). Since this system satisfies (2.12), Corollary 5.7 applies to this system, and we obtain the following result.

THEOREM 5.9. There is no regular dynamic controller using an observation of the form (5.11) that stabilizes the structural acoustics model robustly with respect to delays.

Corollary 5.10. Let $\mathcal{A}$ and $\mathcal{B}$ be as in sections 2,3 , and 4 , and $\mathcal{C}$ be any observation of the form (5.11). Then there does not exist $K \in \mathcal{L}(Y, U)$ such that $\mathcal{A}+\mathcal{B K C}$ generates a $C_{0}$-semigroup in a way that is robustly stable with respect to delays.

Remark 5.11. Note that we have not made any claims about whether one can find an exponentially stabilizing regular dynamic controller in the case where the observation is only taken along the beam and not inside the cavity. We believe that it is unlikely that there is such a stabilizing dynamic controller, but proving this might be difficult: most such lack-of-stabilizability results require either the input operator or observation operator to be bounded, which is not the case here. However, if the control design anticipates small but uncertain delays, then this result shows that no dynamic stabilizer can do the job.

Case 2. Suppose the observation is given by

$$
\mathcal{C} X(t)=\left[v\left(\alpha_{1}\right), \ldots, v\left(\alpha_{j}\right), v_{t}\left(\beta_{1}\right), \ldots, v_{t}\left(\beta_{k}\right), z_{t}\left(x_{i}\right), \ldots, z_{t}\left(x_{l}\right)\right]^{T}
$$

for $\left\{\alpha_{i}\right\}_{i=1}^{j},\left\{\beta_{i}\right\}_{i=1}^{k} \subset \Gamma_{0}$ and $\left\{x_{i}\right\}_{i=1}^{l} \subset \bar{\Omega}$.

As discussed in section 1, since this observation includes point evaluation of acoustic pressure, there is no natural and convenient state space realization; hence we state our results in terms of transfer functions and input-output stabilization. Since $\mathbf{H}(s)=\mathcal{C}_{\Lambda} R(s, A) \mathcal{B}$ and (5.10) holds, it is possible that $\mathbf{H}$ is unstable, but this is not guaranteed, since there might be many pole-zero cancellations. We will take the point of view that if $\mathbf{H}(s)$ is stable, then it can be robustly stabilized by the zero feedback, and focus our attention on the case where $\mathbf{H}$ is unstable.

TheOREM 5.12. Suppose the transfer function $\mathbf{H}$ for the system (2.2) with observation (5.12) is not stable. Suppose further that $\mathbf{H}^{c}$ is well posed and is such that $\mathbf{F}^{0}\left(\mathbf{H}, \mathbf{H}^{c}\right)$ is stable (see Definition 2.5). Then the stability of $\mathbf{F}^{0}\left(\mathbf{H}, \mathbf{H}^{c}\right)$ is not robust with respect to delays. In particular, the conclusions in Theorem 5.6 hold.

Proof. By Theorems 4.6 and 4.8, $\mathbf{H}$ is regular with feedthrough 0. Since $\mathbf{H}^{c}$ is well posed, $\mathbf{H H}^{c}$ is regular with feedthrough 0 . Since $\mathbf{H}$ is analytic in $\overline{\mathbb{C}_{0}}$, the only way it can be unstable is if (5.9) is satisfied. The conclusion follows from Theorem 5.6 .

Remark 5.13. The above results will still be true if $\mathcal{C}$ is replaced by any observation operator such that the open-loop system is regular. We focus on the particular observations (5.11) and (5.12) because point observation of the beam displacement, beam velocity, and acoustic pressure are well motivated physically. 
6. Comparison with numerical results. The feedback control we considered in the previous section (see Figure 5.1) is not the same as the control discussed in [8]. In [8] a noise term $\eta(t)$ and an exogenous forcing function $f(t)$ are included in the plant, and the feedback compensator includes a tracking term. In that paper numerical studies are done on a (necessarily finite dimensional) Galerkin approximation to the system and controller. Among these studies is a look at the effect of delays introduced in three places in the feedback loop for this finite dimensional model. In this section we give some results about the effect of these delays on the full infinitedimensional model for this system. We then give some heuristic explanations for the numerical results obtained in [8] for the finite dimensional model, using a frequency domain analysis.

In [8] the fourth equation in (2.2) is replaced by

$v_{t t}(\xi, t)=-\Delta^{2} v(\xi, t)-\Delta^{2} v_{t}(\xi, t)-z_{t}(\xi, t)+B u(t)+b(\xi) f(t), \quad \xi \in \Gamma_{0}, \quad t \in[0, \infty)$,

where $b$ represents the spatial distribution of the forcing term. In $[8] b(\zeta) \equiv 1$, that is, the forcing term acts the same on all points of the active boundary. We further include the noise term $\mathcal{E} \eta(t)$ in the model, where $\eta(t) \in W$, a Hilbert space, and $\mathcal{E} \in \mathcal{L}(W, \mathcal{X})$. Therefore $(2.13)$ is augmented to

$$
\dot{X}(t)=\mathcal{A} X(t)+\mathcal{B} u(t)+\mathcal{B}_{1} f(t)+\mathcal{E} \eta(t),
$$

where

$$
\mathcal{B}_{1}=[0,0,0, b]^{T} \in \mathcal{X}
$$

Let $\mathcal{C}$ be of the form (5.11) or (5.12), let $p$ be the dimension of the range of $\mathcal{C}$, and let $Y=\mathbb{R}^{p}$. The observation for (6.2) will be formally given by

$$
y(t)=\mathcal{C}_{\Lambda} X(t)+E \eta(t),
$$

where $E \in \mathcal{L}(W, Y)$. We do not worry here about the admissibility of $\mathcal{C}$ for $S(t)$, since we will be doing our robustness analysis in the frequency domain, and that analysis is justified by the work in sections 3 and 4 . Also, we do not have a feedthrough term $D u(t)$ in the observation (6.3) because, as shown in section 4, the feedthrough is zero.

If the initial condition is $X(0)=X_{0}$, the system (6.2), (6.3) can be described in input-output terms as

$$
\hat{y}(s)=\mathbf{H}(s) \hat{u}(s)+\mathbf{H}_{1}(s) \hat{f}(s)+\mathbf{H}_{2}(s) \hat{\eta}(s)+\hat{\nu}(s) ;
$$

here $\nu$ is the observation of the system, depending on the initial data $X_{0}$, but with $u \equiv 0, \eta \equiv 0$, and $f \equiv 0 . \mathbf{H}$ is the transfer function from $u$ to $y$ (analyzed in detail in sections 3 and 4), $\mathbf{H}_{1}$ is the transfer function from $f$ to $y$, and $\mathbf{H}_{2}$ is the transfer function from $\eta$ to $y$. If the observation $\mathcal{C}$ does not contain point observations of acoustic pressure, we can write

$$
\begin{array}{rlrl}
\hat{\nu}(s) & =\mathcal{C}_{\Lambda} R(s, \mathcal{A}) X_{0}, & \mathbf{H}(s) & =\mathcal{C}_{\Lambda} R(s, \mathcal{A}) \mathcal{B}, \\
\mathbf{H}_{1}(s)=\mathcal{C}_{\Lambda} R(s, \mathcal{A}) \mathcal{B}_{1}, & \mathbf{H}_{2}(s)=\mathcal{C}_{\Lambda} R(s, \mathcal{A}) \mathcal{E}+E .
\end{array}
$$




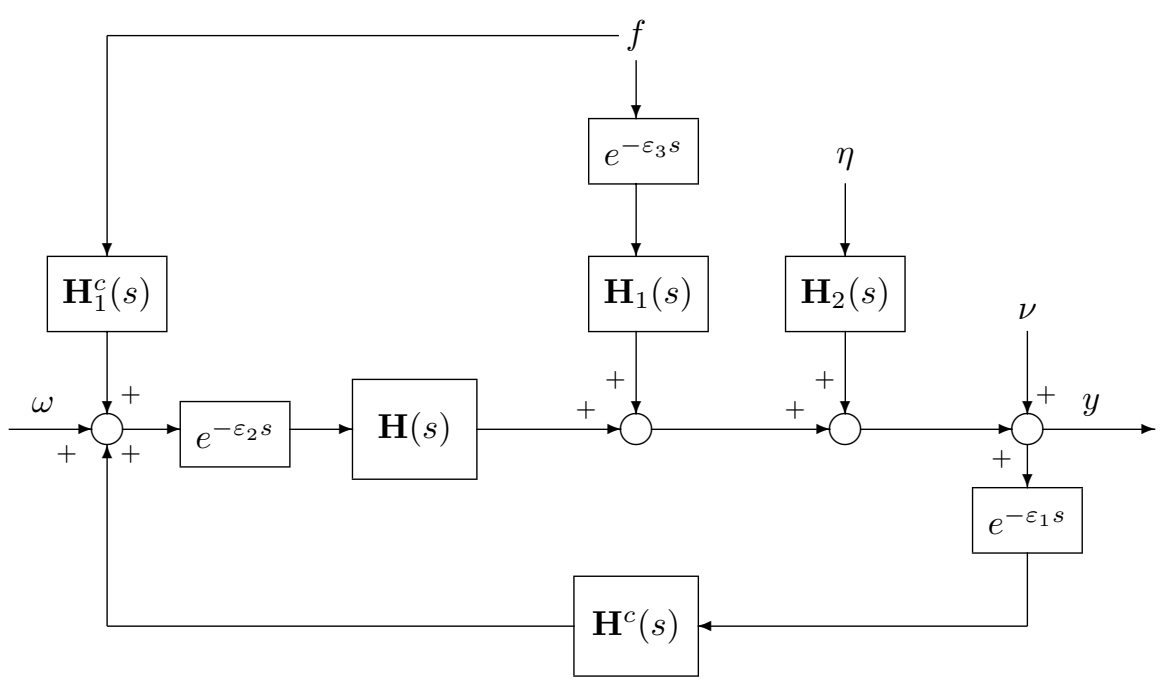

FIG. 6.1. Controller with tracking and delays.

If $\mathcal{C}$ does contain point observations of acoustic pressure, results in sections 3 and 4 show that $\mathbf{H}_{1}$ is regular with feedthrough 0 . In this case, since $\mathcal{C}$ is not even bounded on $\mathcal{D}(\mathcal{A})$, the other transfer functions in (6.5), (6.6) are regular only if $X_{0}$ and $\mathcal{E}$ are sufficiently smooth. We will assume in this section that this is the case.

We will not be concerned about the exact nature of the feedback here, but we will insist that the controller be of the following form in the frequency domain:

$$
\hat{u}(s)=\mathbf{H}^{c}(s) \hat{y}(s)+\mathbf{H}_{1}^{c}(s) \hat{f}(s)+\hat{\omega}(s),
$$

where $\mathbf{H}^{c}$ and $\mathbf{H}_{1}^{c}$ are well-posed transfer functions, $\mathbf{H}_{1}^{c}$ contains any tracking terms, and $\hat{\omega}$ contains initial state information for the compensator. The feedback control in [8] is of this form if the solutions to two algebraic Riccati equations exist and are of sufficient smoothness; it is also of this form if it is a finite dimensional controller computed using the Galerkin approximation to the system. The closed-loop system is shown in Figure 6.1, ignoring the delay blocks.

Solving for $\hat{y}$ in (6.4) and (6.7), we obtain

$$
\hat{y}=\left(I-\mathbf{H H}^{c}\right)^{-1} \mathbf{H}_{2} \hat{\eta}+\left(I-\mathbf{H H}^{c}\right)^{-1}\left(\mathbf{H}_{1}+\mathbf{H} \mathbf{H}_{1}^{c}\right) \hat{f}+\left(I-\mathbf{H H}^{c}\right)^{-1}(\mathbf{H} \hat{w}+\hat{\nu}) .
$$

An obvious design goal for the compensator is to make the transfer function from $\omega$ to $y$ and the transfer function from $\nu$ to $y$ stable, so we assume that $\mathbf{H}^{c}$ has been chosen so that

$$
\left(I-\mathbf{H H}^{c}\right)^{-1} \in H^{\infty} \quad \text { and } \quad\left(I-\mathbf{H H}^{c}\right)^{-1} \mathbf{H} \in H^{\infty} .
$$

As in [8], we consider delays in three places in the feedback loop:

(1) A delay $\varepsilon_{1} \geq 0$ in the plant output $y(t)$;

(2) A delay $\varepsilon_{2} \geq 0$ in the input voltage $u(t)$; and

(3) A delay $\varepsilon_{3} \geq 0$ in the forcing signal $f(t)$. 
These three delays are shown in Figure 6.1. When these delays are taken into account, (6.8) becomes

$$
\begin{aligned}
\hat{y}=(I- & \left.e^{-\left(\varepsilon_{1}+\varepsilon_{2}\right)} \cdot \mathbf{H} \mathbf{H}^{c}\right)^{-1} \mathbf{H}_{2} \hat{\eta}+\left(I-e^{-\left(\varepsilon_{1}+\varepsilon_{2}\right) \cdot} \mathbf{H} \mathbf{H}^{c}\right)^{-1}(\mathbf{H} \hat{w}+\hat{\nu}) \\
& +\left(I-e^{-\left(\varepsilon_{1}+\varepsilon_{2}\right)} \cdot \mathbf{H} \mathbf{H}^{c}\right)^{-1}\left(\varepsilon^{-\varepsilon_{3} \cdot} \mathbf{H}_{1}+\varepsilon^{-\varepsilon_{2}} \cdot \mathbf{H H}_{1}^{c}\right) \hat{f} .
\end{aligned}
$$

In the following results, when we write $\varepsilon_{n}$, we assume that it is not $\varepsilon_{1}, \varepsilon_{2}$, or $\varepsilon_{3}$ as defined above.

Proposition 6.1. Suppose (6.9) holds and $\mathbf{H}$ is not stable. Then there exist sequences $\left\{\varepsilon_{n}\right\}$ and $\left\{p_{n}\right\}$ with

$$
\varepsilon_{n}>0, \quad \varepsilon_{n} \rightarrow 0, \quad p_{n} \in \mathbb{C}_{0}, \quad\left|\operatorname{Im}\left(p_{n}\right)\right| \rightarrow \infty
$$

such that for any $n \in \mathbb{N}, p_{n}$ is a pole of $\left(I-e^{-\left(\varepsilon_{1}+\varepsilon_{2}\right)} \cdot \mathbf{H H}^{c}\right)^{-1}$ when $\varepsilon_{1}+\varepsilon_{2}=\varepsilon_{n}$.

Proof. In sections 3 and 4 we showed that $\mathbf{H}$ is regular with feedthrough 0 . Since $\mathbf{H}^{c}$ is well posed, $\mathbf{H H}^{c}$ is regular with feedthrough 0 . We first need to show that

$$
\limsup _{s \in \mathbb{C}_{0},|s| \rightarrow \infty}\left\|\mathbf{H}(s) \mathbf{H}^{c}(s)\right\|_{\mathcal{L}(Y)}=\infty .
$$

Suppose (6.11) is not true. Then there exist $R, M>0$ such that

$$
\left\|I-\mathbf{H}(s) \mathbf{H}^{c}(s)\right\|_{\mathcal{L}(Y)} \leq M \text { for } s \in \mathbb{C}_{0} \cap\{|s|>R\} .
$$

Note that

$$
\mathbf{H}=\left(I-\mathbf{H H}^{c}\right)\left(I-\mathbf{H} \mathbf{H}^{c}\right)^{-1} \mathbf{H},
$$

so (6.12) and the second equation in (6.9) imply that $\|\mathbf{H}(s)\|_{\mathcal{L}(U, Y)}$ is bounded for $s \in \mathbb{C}_{0} \cap\{|s|>R\}$, which contradicts the hypothesis that $\mathbf{H}$ is unstable and the fact that $\mathbf{H}$ is analytic in $\mathbb{C}_{0}$. Hence (6.11) must be true. Using Lemma 6.3 in [17], we get

$$
\limsup _{s \in \mathbb{C}_{0},|s| \rightarrow \infty} r\left(\mathbf{H}(s) \mathbf{H}^{c}(s)\right)=\infty,
$$

where $r$ denotes the spectral radius. Theorem 5.3 in [17] now implies that there exist sequences $\left\{\varepsilon_{n}\right\}$ and $\left\{p_{n}\right\}$ with

$$
\varepsilon_{n}>0, \quad \varepsilon_{n} \rightarrow 0, \quad p_{n} \in \mathbb{C}_{0}, \quad\left|\operatorname{Im}\left(p_{n}\right)\right| \rightarrow \infty,
$$

such that for any $n \in \mathbb{N}, p_{n}$ is a pole of $\left(I-e^{-\left(\varepsilon_{1}+\varepsilon_{2}\right)} \cdot \mathbf{H} \mathbf{H}^{c}\right)^{-1} \mathbf{H} \mathbf{H}^{c}$ when $\varepsilon_{1}+\varepsilon_{2}=\varepsilon_{n}$. The proposition now follows from the fact that

$$
\left(I-e^{-\left(\varepsilon_{1}+\varepsilon_{2}\right)} \cdot \mathbf{H H}^{c}\right)^{-1}=e^{-\left(\varepsilon_{1}+\varepsilon_{2}\right) \cdot}\left(I-e^{-\left(\varepsilon_{1}+\varepsilon_{2}\right)} \cdot \mathbf{H H}^{c}\right)^{-1} \mathbf{H} \mathbf{H}^{c}+I .
$$

In [8], numerical tests imply that the stability of the closed-loop system can handle small delays $\varepsilon_{3}$ in the forcing function $f(t)$, can tolerate very small delays $\varepsilon_{1}$ in $y(t)$, and becomes unstable for any delays $\varepsilon_{2}$ in $u(t)$. Direct comparison of the lack-of-robustness results in Proposition 6.1 with the results in [8] is difficult, since our results are for the full infinite dimensional system and controller, and the results in [8] are for a finite dimensional approximation of the system and controller, and also 
because the results in [8] are for only one initial state. Nonetheless, we can use our framework to give heuristic explanations for the results from [8].

Since in the envisioned applications $f(t)$ will be periodic, we cannot necessarily expect that $y(t)$ defined by $(6.10)$ is in $L^{2}[0, \infty ; Y]$, even if the transfer functions are stable. However, we will assume that the tracking component of the controller is chosen to minimize the effect of $f$ on $y$. In particular, we assume that $\mathbf{H}_{1}^{c}$, which contains the tracking component of the controller, is chosen so that the term $\left(\mathbf{H}_{1}+\right.$ $\mathbf{H H}_{1}^{c}$ ) in (6.8) has relatively small $H^{\infty}$-norm. First suppose that $\varepsilon_{3}=0$. Both $\varepsilon_{1}$ and $\varepsilon_{2}$ effect $\left(I-e^{-\left(\varepsilon_{1}+\varepsilon_{2}\right)} \cdot \mathbf{H H}^{c}\right)^{-1}$, and $\varepsilon_{2}$ also effects the term $\left(\mathbf{H}_{1}+e^{-\varepsilon_{2} \cdot} \mathbf{H H}_{1}^{c}\right)$ in (6.10). Since $e^{-\varepsilon_{2} s}$ can be far from 1 when $s$ is on the imaginary axis and $|s|$ is large, small $\varepsilon_{2}$ could easily counteract the minimizing effect of $\mathbf{H}_{1}^{c}$ on $\left\|\mathbf{H}_{1}(s)+e^{-\varepsilon_{2} s} \mathbf{H}(s) \mathbf{H}_{1}^{c}(s)\right\|$. Thus, $\varepsilon_{2}$ should have a stronger destabilizing effect on the closed-loop system than $\varepsilon_{1}$, which has exactly the same effect on $\left(I-e^{-\left(\varepsilon_{1}+\varepsilon_{2}\right)} \cdot \mathbf{H} \mathbf{H}^{c}\right)^{-1}$ as $\varepsilon_{2}$ does.

Now suppose that $\varepsilon_{1}=\varepsilon_{2}=0$. The delay $\varepsilon_{3}$ only effects the term $\left(e^{-\varepsilon_{3}} \cdot \mathbf{H}_{1}+\right.$ $\left.\mathbf{H H}_{1}^{c}\right)$ in (6.10), and can easily counteract the effect of $\mathbf{H}_{1}^{c}$ on $\left\|\left(e^{-\varepsilon_{3} s} \mathbf{H}_{1}(s)+\mathbf{H}(s) \mathbf{H}_{1}^{c}(s)\right)\right\|$ for $s$ on the imaginary axis. However, since $\mathcal{B}_{1}$ is quite smooth - for instance, in [8] it is $[0,0,0,1]^{T}$ - it is possible that $\mathbf{H}_{1}(s)=\mathcal{C} R(s, \mathcal{A}) \mathcal{B}_{1}$ is not large when $s$ is on the imaginary axis and $|s|$ is large. This would mitigate the effect of $\varepsilon_{3}$ on $\left(e^{-\varepsilon_{3} \cdot} \mathbf{H}_{1}+\mathbf{H H}_{1}^{c}\right)$.

7. Conclusions. To obtain the results in this paper, we used a combination of techniques. As we have seen in sections 5 and 6 , the effect of small delays on feedback control is best handled using a frequency domain approach. In order to use this approach we first must show that the structural acoustics system under consideration is in the class of regular systems; we can then readily apply results in [17] about delay robustness for regular systems. It is often a challenge to show that a given system is regular. For the structural acoustic model in a rectangular cavity, to prove regularity we used sharp PDE estimates from [1, 2, 3, 4], $C_{0}$-semigroup results from [15], and delicate estimates involving harmonic analysis; for nonrectangular cavities, microlocal analysis is required (see [5]).

Since there is extensive literature on control design for regular systems, the implications of regularity go beyond the study of robustness with respect to delays. For instance, it might be possible to use the regular systems framework to study the effect of additive perturbations on output feedback stabilization [19], to design adaptive control [18], to give Youla parametrizations for stabilizing controllers [27], or to study the relationship between input-output stabilization and exponential stabilization [21, 22].

Acknowledgment. The authors would like to thank Hartmut Logemann for assistance in the proof of Proposition 6.1.

\section{REFERENCES}

[1] G. Avalos, Well-Posedness for a Coupled Hyperbolic/Parabolic System Seen in Structural Acoustics, preprint 1346, Institute for Mathematics and Its Applications, Minneapolis, MN, 1995.

[2] G. Avalos And I. Lasiecka, A differential Riccati equation for the active control of a problem in structural acoustics, J. Optim. Theory Appl., 91 (1996), pp. 695-728.

[3] G. Avalos And I LasieCKa, The strong stability of a semigroup arising from a coupled hyperbolic/parabolic system, Semigroup Forum, 57 (1998), pp. 278-292.

[4] G. Avalos, Sharp regularity estimates for solutions of the wave equation and their traces with prescribed Neumann data, Appl. Math. Optim., 35 (1997), pp. 203-219.

[5] G. Avalos, I. Lasiecka, And R. Rebarber, Well-posedness of a structural acoustics control model with point observation, preprint. 
[6] H.T. Banks, M.A. Demetriou, and R.C. Smith, Robust output feedback control in a 2-D structural acoustic model with piezoceramic actuators, in Proceedings of the 1994 International Conference on Intelligent Materials, Blacksburg, VA, 1994, C.A. Rogers, ed., Technomics, Lancaster, PA, pp. 109-127.

[7] H.T. Banks, M.A. Demetriou and R.C. Smith, An $H^{\infty}$ minmax periodic control in a 2-D structural acoustics model with piezoceramic actuators, IEEE Trans. Automat. Control, 41 (1996), pp. 943-959.

[8] H.T. Banks, M.A. Demitriou, and R.C. Smith, Robustness studies for $H^{\infty}$ feedback control in a structural acoustics model with periodic excitation, Internat. J. Robust and Nonlinear Control, 6 (1996), pp. 453-478.

[9] H.T. Banks, W. Fang, R.J. Silcox, and R.C. Smith, Approximation methods for control of structural acoustics models with piezoceramic actuators, J. Intelligent Material Systems Structures, 4 (1993), pp. 98-116.

[10] H.T. Banks AND R.C. Smith, Models for control in smart material structures, Identification and Control in Systems Governed by Partial Differential Equations, SIAM, Philadelphia, PA, 1993, pp. 26-44.

[11] H.T. BAnks AND R.C. Smith, Well-posedness of a model for structural acoustic coupling in a cavity enclosed by a thin cylindrical shell, J. Math. Anal. Appl., 191 (1995), pp. 1-25.

[12] H.T. BANKs AND R.C. SMith, Feedback control of noise in a 2-D nonlinear structural acoustics model, Discrete Contin. Dynam. Systems, 1 (1995), pp. 119-149.

[13] G. Doetsch, Introduction to the Theory and Application of the Laplace Transform, SpringerVerlag, New York, 1974.

[14] P. Grisvard, Caracterization de quelques espaces d'interpolation, Arch. Rational Mech. Anal., 25 (1967), pp. 40-63.

[15] S.G. Krein, Linear Differential Equations in Banach Space, Transl. Math. Monogr., 29, AMS, Providence, RI, 1971. Translated from the Russian.

[16] I. Lasiecka and R. Triggiani, A cosine operator approach to modeling $L_{2}\left(0, T ; L_{2}(\Gamma)\right)$ boundary input hyperbolic operators, Appl. Math. Optim., 7 (1981), pp. 35-93.

[17] H. Logemann, R. Rebarber, And G. Weiss, Conditions for robustness and nonrobustness of the stability of feedback systems with respect to small delays in the feedback loop, SIAM J. Control Optim., 34 (1996), pp. 572-600.

[18] H. Logemann And S. TOWnLey, Low-gain control of uncertain regular linear systems, SIAM J. Control Optim., 35 (1997), pp. 78-116.

[19] A.J. Pritchard and S. Townley, A Real Stability Radii for Infinite-Dimensional Systems, in Proc. MTNS89, Amsterdam, 1989, pp. 635-646.

[20] J. Prüss, On the spectrum of $C_{0}$-semigroups, Trans. Amer. Math. Soc., 24 (1984), pp. 847-857.

[21] R. Rebarber, Conditions for the equivalence of internal and external stability for distributed parameter systems, IEEE Trans. Automat. Control, 38 (1993) No. 6, pp. 994-998.

[22] R. Rebarber, Exponential stability of coupled beams with dissipative joints: A frequency domain approach, SIAM J. Control Optim., 33 (1995), pp. 1-28.

[23] R. Rebarber And S. Townley, Robustness and continuity of the spectrum for uncertain distributed parameter systems, Automatica, 30 (1995) pp. 1533-1546.

[24] D. Salamon, Realization theory in Hilbert space, Math. Systems Theory, 21 (1989), pp. 147164.

[25] G. WeIss, Transfer functions of regular linear systems, Part I: Characterizations of regularity, Trans. Amer. Math. Soc., 342 (1994), pp. 827-854.

[26] G. WeIss, Regular linear systems with feedback, Math. Control Signals Systems, 7 (1994), pp. $23-57$.

[27] G. Weiss and R.F. Curtain, Dynamic stabilization of regular linear systems, IEEE Trans. Automat. Control, 42 (1997), pp. 4-21.

[28] G. Weiss AND R. REBARBER, Optimizability and estimatability for infinite-dimensional linear systems, preprint.

[29] M. Weiss AND G. WeISS, The spectral factorization approach to the $L Q$ problem for regular linear systems, Proceedings of the 1995 European Control Conference, Rome, Italy. 